\title{
First exploration of the physical Riemann surfaces of the ratio $G_{E}^{\Lambda} / G_{M}^{\Lambda}$
}

\author{
Alessio Mangoni॰ \\ INFN Sezione di Perugia, I-06100 Perugia, Italy \\ Simone Pacetti® \\ INFN Sezione di Perugia and Università di Perugia, I-06100 Perugia, Italy \\ Egle Tomasi-Gustafsson $\odot$ \\ DPhN, IRFU, CEA, Université Paris-Saclay, 91191 Gif-sur-Yvette Cedex, France
}

(Received 22 September 2021; accepted 2 December 2021; published 20 December 2021)

Recently, the BESIII experiment renewed the interest on baryon form factors by measuring the modulus and phase of the ratio $G_{E}^{\Lambda} / G_{M}^{\Lambda}$ between the electric and the magnetic $\Lambda$ form factors with unprecedented accuracy. The BESIII measurement together with older, less precise data, can be analyzed by means of a dispersive procedure based on analyticity and a set of first-principle constraints. Such a dispersive procedure shows the unique ability to determine, for the first time, the complex structure of the ratio knowing its modulus and phase measured by the BESIII Collaboration at only one energy point. Different classes of solutions are obtained, and in all cases, the timelike and spacelike behaviors show interesting properties; spacelike zeros or unexpected large determinations for the phase. More data at different energies would be crucial to enhance the predictive power of the dispersive procedure and to unravel further remarkable features of the $\Lambda$ baryon.

DOI: 10.1103/PhysRevD.104.116016

\section{INTRODUCTION}

The structure of baryons and hence, that of $\Lambda$, can be studied through fundamental quantities called form factors (FFs) (see Ref. [1] for an exhaustive review). They are Lorentz scalar functions depending on the four-momentum squared related to the baryon four-current, and contain pivotal information on their dynamics, as well as on some static properties.

In particular the $\Lambda \bar{\Lambda} \gamma$ vertex is described by two independent FFs, usually represented by the so-called Sachs electric and magnetic FFs, $G_{E}$ and $G_{M}$ [2]. In the Breit frame, which is the reference system where there is no energy exchange, i.e., where the four-momentum of the virtual photon coincides with its three-momentum, the Sachs FFs represent the Fourier transforms of the electric charge and magnetic-momentum spatial distributions of the $\Lambda$ baryon. The study of their ratio represents a crucial point, since the relative phase between the electric and magnetic FFs of baryons, i.e., $\arg \left(G_{E}^{\Lambda} / G_{M}^{\Lambda}\right)$, is directly related to their polarization.

Published by the American Physical Society under the terms of the Creative Commons Attribution 4.0 International license. Further distribution of this work must maintain attribution to the author(s) and the published article's title, journal citation, and DOI. Funded by SCOAP ${ }^{3}$.
The main goals of the present study are the definition and the exploitation of a dispersive approach, based on first principles and theoretical constraints, to analyze the available data on the ratio $G_{E}^{\Lambda} / G_{M}^{\Lambda}$. A unique and novel outcome of such analysis is the complete disclosure of the complex structure of the FF ratio. In fact, besides the modulus and the phase, the procedure is able to establish the specific determination of the phase itself, i.e., the Riemann surface to which the physical values of the ratio belong.

\section{THE SPECIAL CASE OF $\Lambda$ BARYONS}

The $\Lambda$ FFs, like all baryon FFs, are Lorentz scalar functions of the four-momentum transfer squared $q^{2}$, analytic in the $q^{2}$-complex plane with a branch cut along the positive real axis from the theoretical threshold, $q^{2}=$ $q_{\mathrm{th}}^{2}$ up to infinity. The theoretical threshold corresponds to the mass squared of the lightest hadronic channel that can couple with the virtual photon and with the $\Lambda \bar{\Lambda}$ state. In the case of nucleons, such a hadronic state is that of the two charged pions $\pi^{+} \pi^{-}$and hence the theoretical threshold is $\left(2 M_{\pi}\right)^{2} ; M_{\pi}$ being the charged pion mass.

However, since the $\Lambda$ baryon and hence also the $\Lambda \bar{\Lambda}$ system have isospin zero, the theoretical threshold of the $\Lambda$ FFs is $q_{\mathrm{th}}^{2}=\left(2 M_{\pi}+M_{\pi^{0}}\right)^{2}$. Indeed the lightest isoscalar, i.e., having isospin zero, hadronic state is that of three pions: $\pi^{+} \pi^{-} \pi^{0}$. 
The only portion of the $q^{2}$-complex plane that is experimentally accessible is the real axis; indeed, only real values of $q^{2}$ can be tested in laboratories.

Due to the Hermitian properties of the electromagnetic current operator of the baryon vertex $\Lambda \bar{\Lambda} \gamma$, the FFs that parametrize such a current operator are real at real values of $q^{2}$ belonging to the analyticity domain, namely $q^{2} \leq q_{\mathrm{th}}^{2}$, and in general verify the Schwarz reflection principle; $G\left(q^{2 *}\right)=G^{*}\left(q^{2}\right)$, with $G=G_{E}^{\Lambda}, G_{M}^{\Lambda}$. It follows that, on the edges of the branch cut $\left(q_{\mathrm{th}}^{2}, \infty\right)$, they are complex and have a nonvanishing imaginary part. In fact, at $z=q^{2}+i \epsilon$, with $q^{2}>q_{\mathrm{th}}^{2}$ and $\epsilon \rightarrow 0^{+}$, the imaginary part is

$$
\begin{aligned}
\operatorname{Im}[G(z)] & =\frac{G(z)-G^{*}(z)}{2 i}=\frac{G\left(q^{2}+i \epsilon\right)-G^{*}\left(q^{2}+i \epsilon\right)}{2 i} \\
& =\frac{G\left(q^{2}+i \epsilon\right)-G\left(q^{2}-i \epsilon\right)}{2 i} \neq 0,
\end{aligned}
$$

where the inequality follows from the discontinuity of the FFs across the branch cut.

In summary, FFs are real for real values of $q^{2}$ below the theoretical threshold, i.e., at $q^{2} \leq q_{\mathrm{th}}^{2}$, and hence in the whole spacelike region, $q^{2} \leq 0$, together with the timelike interval $\left(0, q_{\mathrm{th}}^{2}\right)$, while they are complex in the remaining part of the timelike region, namely for $q^{2} \geq q_{\mathrm{th}}^{2}$.

In principle, even in an ideal laboratory without any experimental limits, FFs would not be completely accessible in the entire kinematic region, that is $q^{2} \in(-\infty, \infty)$.

Form factor values can be extracted from specific observables in each kinematic region.

(a) In the spacelike region, $q^{2} \leq 0, G_{E}^{\Lambda}$ and $G_{M}^{\Lambda}$ are real and their values can be extracted by the differential cross section of the scattering reaction $e^{-} \Lambda \rightarrow e^{-} \Lambda$. The Feynman diagram of the reaction is shown on the left side of Fig. 1, lying in the corresponding kinematical region.

(b) In the timelike range $q^{2}>q_{\text {phy }}^{2}$, where $q_{\text {phy }}^{2}=\left(2 M_{\Lambda}\right)^{2}$ is the physical or production threshold where the FFs have complex values, their moduli can be extracted from the differential cross section of the annihilation processes $e^{+} e^{-} \leftrightarrow \Lambda \bar{\Lambda}$. Also, in this case, the Feynman diagram of the reaction positioned in the corre-

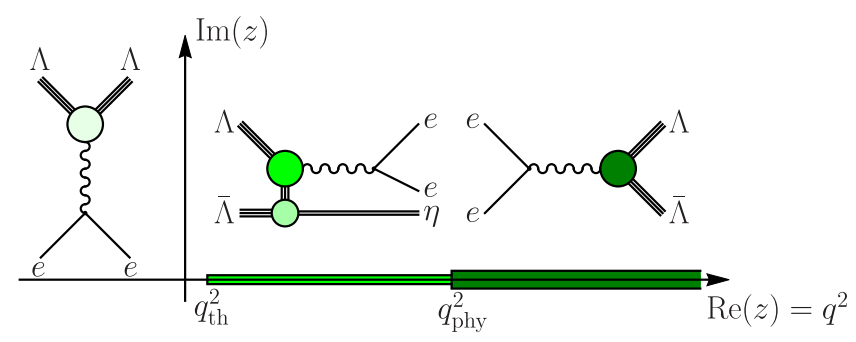

FIG. 1. Schematic representation of $z=q^{2}$-complex plane, with the Feynman diagrams, at the lowest order of the reactions that allow us to extract FF values in each kinematic region. sponding kinematical region is shown on the right side of Fig. 1. Moreover, the relative phase $\arg \left(G_{E}^{\Lambda} / G_{M}^{\Lambda}\right)$ can be extracted from the measurement of the polarization of one of the outgoing baryons along the direction orthogonal to the scattering plane.

(c) In the so-called unphysical region, i.e., between the theoretical and the physical threshold, at $q_{\mathrm{th}}^{2}<$ $q^{2} \leq q_{\text {phy }}^{2}$, moduli of the FFs, can in principle, be extracted from the differential cross section of the reaction $\Lambda \bar{\Lambda} \rightarrow \eta e^{+} e^{-}$, where the $\eta$ meson is emitted by one of the initial baryons. The corresponding Feynman diagram is shown in the middle of Fig. 1. The possibility of using this process to experimentally access the unphysical region has been deeply investigated in the case of proton FFs, for the corresponding reaction $p \bar{p} \rightarrow e^{+} e^{-} \pi^{0}[3,4]$.

The present unavailability of a stable target and, a fortiori, of stable beams of $\Lambda$ baryons, prevents the possibility of making scattering and $\Lambda \bar{\Lambda}$ annihilation experiments. It follows that, $\Lambda$ FFs cannot be measured either in the spacelike region or in the unphysical region, as can be done in the case of stable baryons; namely, for proton and neutron. On the other hand, the weak decay $\Lambda \rightarrow p \pi^{-}$, commonly exploited to identify the $\Lambda$ baryon itself (thanks to the charged hadrons in the final state) has the crucial advantage of being self-analyzing, meaning that the $\Lambda$ polarization can be extracted from the angular distribution of the final proton. In light of this, it is possible to access the relative phase $\arg \left(G_{E}^{\Lambda} / G_{M}^{\Lambda}\right)$ without the need of direct polarization measurements that would require inserting polarimeters in the main detector; consequently worsening its general tracking performances.

\section{POLARIZATION OBSERVABLES}

It is well known [5] that, even though the initial leptons are unpolarized, the baryon $B$ and the antibaryon $\bar{B}$ produced by the annihilation reaction $e^{+} e^{-} \rightarrow B \bar{B}$ are polarized along the direction orthogonal to the scattering plane; namely, the plane containing the three-momenta of the initial and final particles in the $e^{+} e^{-}$center of mass frame. This spontaneous polarization is due to the complex nature of the FFs and hence it can be used to measure their complexity. In particular, the $y$-component of the polarization vector of the outgoing baryon $B$, defining the $x z$ plane as the scattering plane, has the expression [5]

$$
\mathcal{P}_{y}=-\frac{\sqrt{\frac{q^{2}}{4 M_{B}^{2}}} \frac{\left|G_{E}^{\Lambda}\right|}{\left|G_{M}^{\Lambda}\right|} \sin (2 \theta) \sin \left(\arg \left(\frac{G_{E}^{\Lambda}}{G_{M}^{\Lambda}}\right)\right)}{\frac{q^{2}}{4 M_{B}^{2}}\left(1+\cos ^{2}(\theta)\right)+\frac{\left|G_{E}^{\Lambda}\right|^{2}}{\left|G_{M}^{\Lambda}\right|^{2}} \sin ^{2}(\theta)},
$$

where $q$ is the transfer four-momentum, $M_{B}$ is the baryon mass and $\theta$ is the scattering angle in the $e^{+} e^{-}$center of mass frame. It is evident that the $y$ polarization vanishes in 
case of relative reality of FFs, i.e., if the relative phase $\arg \left(G_{E}^{\Lambda} / G_{M}^{\Lambda}\right)$ is an integer multiple of $\pi$ radians.

On the other hand, the polarization is maximum at

$$
\cos (\theta)= \pm \sqrt{\frac{q^{2} /\left(4 M_{B}^{2}\right)+\left|G_{E}^{\Lambda}\right|^{2} /\left|G_{M}^{\Lambda}\right|^{2}}{3 q^{2} /\left(4 M_{B}^{2}\right)+\left|G_{E}^{\Lambda}\right|^{2} /\left|G_{M}^{\Lambda}\right|^{2}}}
$$

close to the production threshold, i.e., at $q^{2} \simeq\left(2 M_{B}\right)^{2}$, and assuming $\left|G_{E}^{\Lambda}\right| \simeq\left|G_{M}^{\Lambda}\right|$, gives $\cos (\theta) \simeq \pm 1 / \sqrt{2}$, and hence $\theta \simeq \pi / 4+k \pi, 3 \pi / 4+k \pi$, with $k \in \mathbb{Z}$.

In the light of this remark, the value of the sinus of the relative phase $\arg \left(G_{E}^{\Lambda} / G_{M}^{\Lambda}\right)$ at a given $q^{2}$ can be extracted from the experimental data on the polarization component $\mathcal{P}_{y}$, measured at that $q^{2}$ and at any $\theta$. Indeed, the relative phase, like the FFs themselves, depends solely on the fourmomentum transferred squared.

Finally, it is interesting to notice that the knowledge of the sinus does not allow us to have any clue on the determination of the relative phase whatever information it does contain.

\section{DATA}

At present, only two sets of data, both of them on the modulus and the phase of the ratio $G_{E}^{\Lambda} / G_{M}^{\Lambda}$, are available. They have been obtained by the BABAR experiment [6] in 2006 and, more recently, in 2019, by the BESIII experiment [7]. The $B A B A R$ data set consists of two data points on the modulus and one point, obtained by collecting the whole statistics, on the phase of the ratio $G_{E}^{\Lambda} / G_{M}^{\Lambda}$. The low statistics of the BABAR measurement is a consequence of the initial-state-radiation technique, that has to be used by fixed-energy machines to reproduce the same observables available at a typical $e^{+} e^{-}$machine with energy scan.

The data from the BESIII experiment consists of the modulus and phase of the ratio $G_{E}^{\Lambda} / G_{M}^{\Lambda}$ at a unique energy point. Nevertheless, the achieved level of accuracy is quite high because it was a direct measurement, obtained by collecting events $e^{+} e^{-} \rightarrow \Lambda \bar{\Lambda}$ at the desired energy.

All BABAR and BESIII data on the ratio $G_{E}^{\Lambda} / G_{M}^{\Lambda}$ are shown in Fig. 2; in particular, from left to right, the modulus, the phase and the sinus of the phase, which actually represents the genuine experimental observable extracted from the $y$-component of the $\Lambda$ polarization vector [see Eq. (1)].

As already highlighted, this implies that the determination of the phase $\mathrm{e}^{1}$ is not experimentally accessible through the polarization measurement only.

\footnotetext{
${ }^{1}$ At a given value $x=\sin (\alpha) \in[-1,1]$ of the sinus do correspond infinite angles, namely $\alpha_{k}=\arcsin (x)+2 k \pi$ and $\alpha_{k}^{\prime}=-\arcsin (x)+(2 k+1) \pi$, for all $k \in \mathbb{Z}$, with $\arcsin (x) \in$ $[-\pi / 2, \pi / 2)$.
}
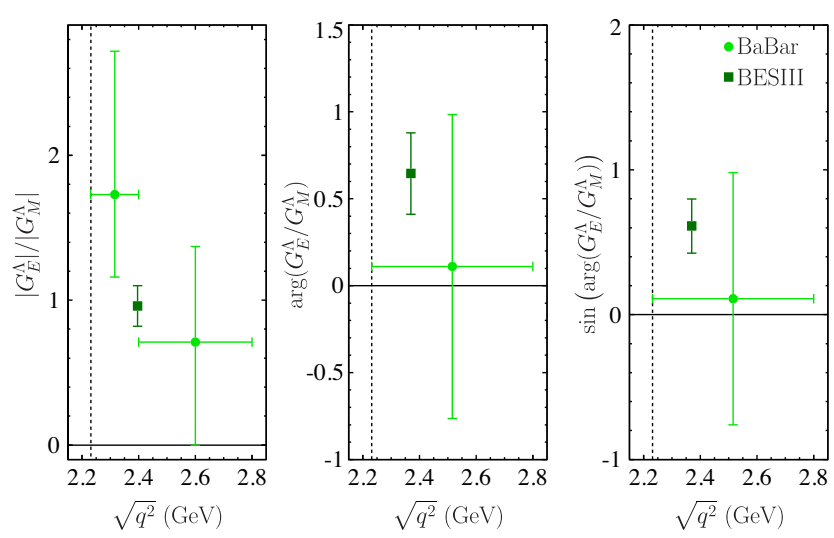

FIG. 2. Data on the modulus (left panel) the phase (central panel) and the sinus of the phase (right panel) of the ratio $G_{E}^{\Lambda} / G_{M}^{\Lambda}$ measured by BABAR [6] (light green solid circles) and BESIII [7] (dark green solid square) experiments.

\section{ON THE MEANING OF THE PHASE DETERMINATION}

In order to understand the meaning and hence the information that is embodied in the phase of an analytic multivalued function, and in particular in its determination, we study in some detail the following example. Consider an analytic multivalued function $R(z)$, defined in the domain $D=\left\{z: z \notin\left(x_{0}, \infty\right) \cup\left\{p_{j}\right\}_{j=1}^{N^{\prime}}\right\}$, having the real branch cut $\left(x_{0}, \infty\right) \subset \mathbb{R}$ and the set of zeros $\left\{z_{k}\right\}_{k=1}^{M^{\prime}} \subset \mathbb{C}$, where $\left\{m_{k}\right\}_{k=1}^{M^{\prime}} \subset \mathbb{N}$ is the set of the corresponding orders, while $\left\{p_{j}\right\}_{j=1}^{N^{\prime}} \subset \mathbb{C}$ and $\left\{n_{j}\right\}_{j=1}^{N^{\prime}} \subset \mathbb{N}$ are the sets of the poles and of the corresponding orders. Figure 3 shows a pictorial view of the domain $D$, the branch cut is highlighted in light

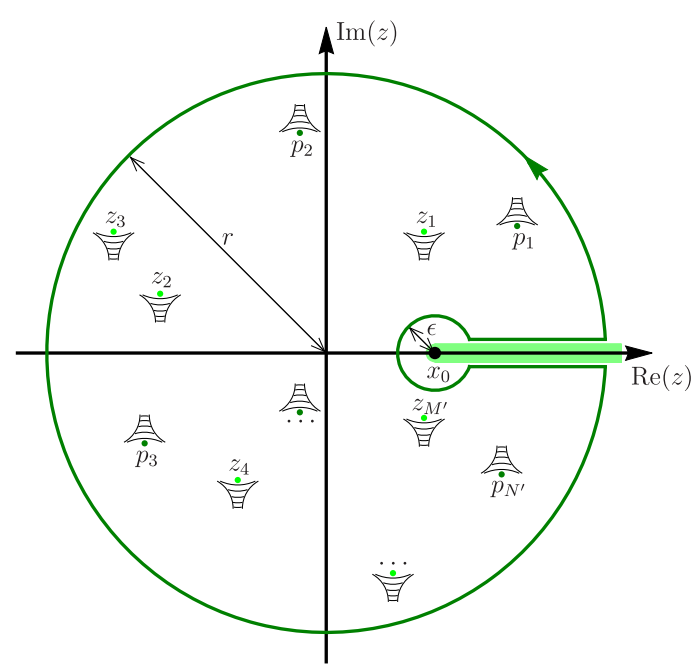

FIG. 3. The dark green curve represents the $\Gamma_{r, \epsilon}$ integration path, while the dark and light green solid circles indicate the poles and zeros surrounded by $\Gamma_{r, \epsilon}$. The real and positive branch cut $\left(x_{0}, \infty\right)$ is highlighted by a light-green band. 
green, while zeros and poles are indicated by dips and spikes, respectively.

The integral of the logarithmic derivative of the function $R(z) /(2 i \pi)$ over the closed path $\Gamma_{r, \epsilon}$, shown as a dark green curve in Fig. 3, which does not intersect neither poles, nor zeros, having

$$
\begin{aligned}
& r>\max _{\substack{j=1,2, \ldots, N^{\prime} \\
k=1,2, M^{\prime}}}\left\{\left|p_{j}\right|,\left|z_{k}\right|\right\}, \\
& \epsilon<\min _{\substack{j=1,2, \ldots, N^{\prime} \\
k=1,2, \ldots, M^{\prime}}}\left\{\left|x_{0}-p_{j}\right|,\left|x_{0}-z_{k}\right|\right\},
\end{aligned}
$$

where $r$ and $\epsilon$ are the radii of the circles centered at the origin, and in $x_{0}$ gives the difference between the total numbers of zeros $M$ and poles $N$ of the function $R(z)$, lying inside the contour $\Gamma_{r, \epsilon}$. Zeros and poles are counted as many times as their multiplicities, i.e.,

$$
\sum_{k=1}^{M^{\prime}} m_{k}=M, \quad \sum_{j=1}^{N^{\prime}} n_{j}=N .
$$

It follows that

$$
\frac{1}{2 i \pi} \oint_{\Gamma_{r, \epsilon}} \frac{d \ln [R(z)]}{d z} d z=M-N
$$

this is also called the argument principle or Cauchy's argument principle [8].

Assuming that $z=\infty$ is an accumulation point neither of zeros nor of poles of the function $R(z)$, the result of Eq. (2) also holds under the limits $\epsilon \rightarrow 0^{+}$and $r \rightarrow \infty$, so that

$$
\lim _{r \rightarrow \infty} \lim _{\epsilon \rightarrow 0^{+}} \frac{1}{2 i \pi} \oint_{\Gamma_{r, \epsilon}} \frac{d \ln [R(z)]}{d z} d z=M-N .
$$

Moreover, if the function $R(z)$ has the asymptotic behavior suitable to make infinitesimal the contributions of the two $\operatorname{arcs}$

$$
\begin{aligned}
& \gamma_{\epsilon}=\left\{z:\left|z-x_{0}\right|=\epsilon e^{i \phi}, \phi \in(\eta, 2 \pi-\eta), \eta \rightarrow 0^{+}\right\}, \\
& \gamma_{r}=\left\{z:|z|=r e^{i \phi}, \phi \in(\eta, 2 \pi-\eta), \eta \rightarrow 0^{+}\right\},
\end{aligned}
$$

as $\epsilon \rightarrow 0^{+}$and $r \rightarrow \infty$, respectively, i.e.,

$$
\begin{aligned}
& \lim _{\epsilon \rightarrow 0^{+}} \frac{1}{2 i \pi} \oint_{\gamma_{\epsilon}} \frac{d \ln [R(z)]}{d z} d z=0, \\
& \lim _{r \rightarrow \infty} \frac{1}{2 i \pi} \oint_{\gamma_{r}} \frac{d \ln [R(z)]}{d z} d z=0,
\end{aligned}
$$

then the limit of the integral on the left-hand side of Eq. (3) becomes

$$
\begin{aligned}
& \frac{1}{2 i \pi}\left(\int_{x_{0}+i \epsilon}^{\infty+i \epsilon} \frac{d \ln [R(z)]}{d z} d z\right. \\
& \left.\quad-\int_{x_{0}-i \epsilon}^{\infty-i \epsilon} \frac{d \ln [R(z)]}{d z} d z\right)=M-N .
\end{aligned}
$$

Taking advantage from the Schwarz reflection principle [8] $R(z)=R^{*}\left(z^{*}\right)$ and using the polar form $R(z)=$ $|R(z)| e^{i \arg (R(z))}$, the second integral can be written in Cartesian coordinates, i.e., as the combination of its real and imaginary part and it reads

$$
\begin{aligned}
\int_{x_{0}-i \epsilon}^{\infty-i \epsilon} \frac{d \ln [R(z)]}{d z} d z= & \int_{x_{0}}^{\infty} \frac{d \ln [R(x-i \epsilon)]}{d x} d x \\
= & \int_{x_{0}}^{\infty} \frac{d \ln \left[R^{*}(x+i \epsilon)\right]}{d x} d x \\
= & \int_{x_{0}}^{\infty} \frac{d \ln |R(x+i \epsilon)|}{d x} d x \\
& -i \int_{x_{0}}^{\infty} \frac{d \arg (R(x+i \epsilon))}{d x} d x
\end{aligned}
$$

Finally, by using this expression in Eq. (4), it reduces to

$$
\frac{1}{\pi} \int_{x_{0}}^{\infty} \frac{d \arg (R(x+i \epsilon))}{d x} d x=M-N
$$

giving, after the integration of the left-hand side,

$$
\arg (R(\infty))-\arg \left(R\left(x_{0}\right)\right)=\pi(M-N) .
$$

It represents the version of Levinson's theorem [9] which will be extensively used in the following.

Notice that, in the perspective of using the result of Eq. (5) for the ratio of the FFs (which is a function of $q^{2}$ ) the infinitesimal imaginary part $\epsilon$ appearing in the argument of the function $R(z)$ has been omitted, because, as is usually done, the timelike values of the FFs at $q^{2}>q_{\mathrm{th}}^{2}$ are defined as those on the upper edge of the branch cut, i.e., for $q^{2}>q_{\mathrm{th}}^{2}, G_{E, M}^{\Lambda}\left(q^{2}\right) \equiv G_{E, M}^{\Lambda}\left(q^{2}+i \epsilon\right)$.

\section{THE SPECIFIC CASE OF $G_{E}^{\Lambda} / G_{M}^{\Lambda}$}

The multivalued analytic function under consideration is the FF ratio $R\left(q^{2}\right)=G_{E}^{\Lambda}\left(q^{2}\right) / G_{M}^{\Lambda}\left(q^{2}\right)$, whose analytic properties are driven from those of the single electric $G_{E}^{\Lambda}$ and magnetic $G_{M}^{\Lambda}$ FFs.

(a) Analyticity domain. Since, as discussed in Sec. II, both FFs have the same analyticity domain, that is, the $q^{2}$ complex plane with the real and positive (timelike) branch cut $\left(q_{\mathrm{th}}^{2}, \infty\right)$, the ratio is a multivalued meromorphic function [8], having the same branch cut $\left(q_{\mathrm{th}}^{2}, \infty\right)$ and a set of isolated poles corresponding to the zeros of the magnetic FF $G_{M}^{\Lambda}$, that are not canceled 
by equal or higher-order zeros of the electric $\mathrm{FF} G_{E}^{\Lambda}$. However, we assume that the magnetic FF has no zeros, so that the analytic domain of the ratio corresponds to that of the FFs, i.e., $D_{\mathrm{FF}}=\left\{q^{2}: q^{2} \notin\right.$ $\left.\left(q_{\mathrm{th}}^{2}, \infty\right)\right\}$. Even though this is an assumption, it is supported by quite convincing phenomenological and experimental facts. From the phenomenological point of view, there are various acceptable descriptions and parametrizations of the FFs [10,11], which are valid in the whole kinematic region, namely spacelike and timelike values of $q^{2}$. They are based on unitarity and analyticity and are conceived under the assumption of no zeros for $G_{M}\left(q^{2}\right)$. Their success in describing practically all the available data can be taken as a compelling piece of evidence of the goodness of the initial assumption.

On the other hand, the experimental results for the nucleon magnetic FFs, for instance, the most investigated in spacelike and timelike regions, simply because they represent the dominant contribution to the scattering and annihilation cross section at high $\left|q^{2}\right|$, do not show any zero. Although the data refer unavoidably to a limited energy range, such a range is however quite wide, so that, eventually zeros escaping the experimental investigation by lying at high $\left|q^{2}\right|$, would have only negligible effects at low energies where our analysis is focused. Moreover, the ratio has zeros in the same points and of the same order as the electric FF $G_{E}^{\Lambda}$. It follows that, having the normalizations at $q^{2}=0$

$$
\begin{aligned}
G_{E}^{\Lambda}(0) & =Q_{\Lambda}=0 \\
G_{M}^{\Lambda}(0) & =\mu_{\Lambda}=-0.613 \pm 0.004 \mu_{N},
\end{aligned}
$$

where $\mu_{N}=e \hbar /\left(2 M_{p} c\right)$ is the nuclear magneton [12] and, $Q_{\Lambda}$ and $\mu_{\Lambda}$ are the electric charge and magnetic moment of the $\Lambda$ baryon, the ratio has at least a zero in the origin as a consequence of the neutrality of the $\Lambda$ baryon itself.

(b) Complexity. The ratio, as well as the FFs, fulfill the Schwarz reflection principle, $R^{*}\left(q^{2}\right)=R\left(q^{2 *}\right)$, and hence it assumes real values at each real $q^{2}$ belonging to its analyticity domain, i.e.,

$$
R\left(q^{2}\right) \in \mathbb{R}, \quad \forall q^{2} \in D_{\mathrm{FF}} \cap \mathbb{R}=\left(-\infty, q_{\mathrm{th}}^{2}\right) .
$$

On the other hand, it can have a non-null imaginary part at $q_{+}^{2}=q^{2}+i \epsilon$, with $q^{2}>q_{\text {th }}^{2}$ and $\epsilon \rightarrow 0^{+}$; that is, on the upper edge of the timelike branch cut. As discussed in Appendix A, such an imaginary part represents the source of discontinuity across the branch cut. Indeed, passing through the cut, e.g., from $q_{+}^{2}=q^{2}+i \epsilon$ to $q_{-}^{2}=q^{2}-i \epsilon\left(q^{2}>q_{\mathrm{th}}^{2}\right.$ and $\left.\epsilon \rightarrow 0^{+}\right)$, the imaginary part changes sign, while the real part does not vary as it is continuous, ${ }^{2}$

$$
\begin{aligned}
& \lim _{\epsilon \rightarrow 0^{+}} \operatorname{Re}\left(R\left(q^{2}+i \epsilon\right)\right)=\lim _{\epsilon \rightarrow 0^{+}} \operatorname{Re}\left(R\left(q^{2}-i \epsilon\right)\right), \\
& \lim _{\epsilon \rightarrow 0^{+}} \operatorname{Im}\left(R\left(q^{2}+i \epsilon\right)\right)=-\lim _{\epsilon \rightarrow 0^{+}} \operatorname{Im}\left(R\left(q^{2}-i \epsilon\right)\right) .
\end{aligned}
$$

(c) Behavior at the theoretical threshold. Under the assumption of a magnetic FF having no zeros, at the theoretical threshold $q_{\mathrm{th}}^{2}$ the ratio has the same behavior as the electric FF. In particular, following the discussion developed in Appendix A, at this threshold, corresponding to the branch point of the cut, the electric and magnetic FFs behave like the powers

$$
G_{E, M}^{\Lambda}\left(q^{2}\right) \underset{q^{2} \rightarrow q_{\mathrm{th}}^{2}}{\propto}\left(q^{2}-q_{\mathrm{th}}^{2}\right)^{\gamma_{E, M}},
$$

with $\operatorname{Re}\left(\gamma_{E, M}\right)>-1$. Moreover, since the magnetic FF has no zeros, the real part of the power $\gamma_{M}$ can not be greater than or equal to one so it must fulfill the condition: $-1<\operatorname{Re}\left(\gamma_{M}\right) \leq 0$. Therefore, the behavior of the ratio at the theoretical threshold fulfills the requirement of the dispersion relations (DRs), i.e.,

$$
R\left(q^{2}\right) \underset{q^{2} \rightarrow q_{\mathrm{th}}^{2}}{\propto}\left(q^{2}-q_{\mathrm{th}}^{2}\right)^{\gamma_{E}-\gamma_{M}},
$$

with $\operatorname{Re}\left(\gamma_{E}-\gamma_{M}\right)>-1$, as follows from the limitations $\operatorname{Re}\left(\gamma_{E}\right)>-1$ and $-\operatorname{Re}\left(\gamma_{M}\right) \geq 0$. We would like to stress that the one due to the three-pion channel is the only branch cut that has been explicitly introduced. In fact, it contains all the other cuts related to the infinite tower of intermediate processes which can contribute to the FFs. In fact, since the branch cuts start at the on-shell mass squared of the corresponding intermediate states and go up to infinity along the real axis, they represent the sequence of self-contained sets

$$
\begin{gathered}
\underbrace{\left(q_{\mathrm{th}}^{2}, \infty\right)}_{\pi^{+} \pi^{-} \pi^{0} \text { cut }} \underbrace{\left(M_{\omega}^{2}, \infty\right)}_{\omega \text { cut }} \supset \underbrace{\left(\left(M_{\rho}+M_{\pi}\right)^{2}, \infty\right)}_{\rho \pi \text { cut }} \\
\supset \underbrace{\left(4 M_{K}^{2}, \infty\right)}_{K^{+} K^{-} \text {cut }} \supset \underbrace{\left(4 M_{K^{0}}^{2}, \infty\right)}_{K_{S}^{0} K_{L}^{0} \text { cut }} \supset \cdots,
\end{gathered}
$$

and as the obvious consequence of the ordering

$$
q_{\mathrm{th}}^{2}<M_{\omega}^{2}<\left(M_{\rho}+M_{\pi}\right)^{2}<4 M_{K}^{2}<4 M_{K^{0}}^{2}<\cdots .
$$

Formally, by implementing the possibility of having the widest branch cut, i.e., that of the three pions, our procedure of forcing the theoretical and the experimental constraints, that embody the dynamics of all other intermediate channels, should be able to 


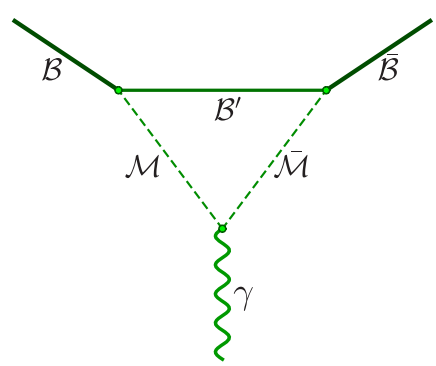

FIG. 4. The $\mathcal{B} \mathcal{M B}^{\prime}$ triangle contribution to the $\mathcal{B}$ baryon FFs.

reproduce the complete discontinuity structure of the FF ratio. These intermediate processes are characterized by the production and annihilation of virtual single multiparticle states, having the quantum numbers of the baryon-antibaryon final state. In the spirit of making a model-independent study, no predefined spectral functions related to specific intermediate states, e.g., as in the case of nucleons $[13,14]$, have been used. Indeed, only theoretical constraints, based on the first principles concepts are considered. Also, the choice of the observable, namely the ratio of the FFs, is crucial in this perspective. In fact, the dominant contributions, such as those due to the opening of production thresholds, lying in the (unexplored) unphysical region have to play similar roles in both electric and magnetic FFs, to which they contribute through the common term $F_{1}$, the Dirac FF. In this way their effects cancel out in the ratio, which should behave like a smooth function of $q^{2}$.

The contribution to the generic $\mathcal{B}$ baryon FFs due to the intermediate processes with the triangular Feynman diagram shown in Fig. 4, where the virtual photon couples with the $\mathcal{M} \overline{\mathcal{M}}$ meson-antimeson pair, which produces the $\mathcal{B} \overline{\mathcal{B}}$ baryon-antibaryon final state by exchanging a further baryon $\mathcal{B}^{\prime}$, may generate an anomalous theoretical threshold $[13,15]$. Such a threshold, representing a branch point, i.e., the opening of a branch cut crucial for the dispersion relations' procedure, could lie below the standard multipion threshold. It follows that it has to be considered in exploiting the analytic continuation technique based on dispersive approaches. The necessary kinematic condition for having this kind of contribution is $[13,15]$

$$
M_{\mathcal{B}}^{2}>M_{\mathcal{B}^{\prime}}^{2}+M_{\mathcal{M}}^{2},
$$

and the corresponding branch point lies at

$$
q_{\mathcal{B}_{\mathcal{M}} \mathcal{B}^{\prime}}^{2}=4 M_{\mathcal{M}}^{2}-\frac{\left(M_{\mathcal{B}}^{2}-M_{\mathcal{B}^{\prime}}^{2}-M_{\mathcal{M}}^{2}\right)^{2}}{M_{\mathcal{B}^{\prime}}^{2}} .
$$

Of course, the FF of the proton, being the lightest baryon, does not have this contribution. Also for the neutron, such an intermediate process is not kinematically allowed. In fact, even considering the lightest meson, i.e., the pion, the inequality of Eq. (8) does not hold, having instead

$$
M_{n}^{2}<M_{p}^{2}+M_{\pi}^{2}
$$

The case of the baryon $\Sigma^{+}\left(\mathcal{B}=\Sigma^{+}\right)$is very interesting because by considering $\mathcal{B}^{\prime}=\Lambda$ and $\mathcal{M}=\pi^{+}$the inequality of Eq. (8) is satisfied and the anomalous threshold from Eq. (9) in units of the pion square mass is

$$
\frac{q_{\Sigma^{+} \pi^{+} \Lambda}^{2}}{M_{\pi}^{2}}=4-\frac{\left(M_{\Sigma}^{2}-M_{\Lambda}^{2}-M_{\pi}^{2}\right)^{2}}{4 M_{\pi}^{2} M_{\Lambda}^{2}} \simeq 3.07 .
$$

It lies below the standard theoretical threshold $q^{2}=$ $4 M_{\pi}^{2}$ so that the branch cut must be enlarged by shifting backwards just at $q_{\Sigma^{+} \pi^{+} \Lambda}^{2}$ the first branch point.

In the case under consideration, i.e., $\mathcal{B}=\Lambda$, we have to consider two triangular contributions with: $\left(\mathcal{B}^{\prime}, \mathcal{M}\right)=\left(p, K^{+}\right)$and $\left(\mathcal{B}^{\prime}, \mathcal{M}\right)=\left(n, K^{0}\right)$. In both cases the kinematical constraint is verified

$$
M_{\Lambda}^{2}>M_{p}^{2}+M_{K}^{2}, \quad M_{\Lambda}^{2}>M_{n}^{2}+M_{K^{0}}^{2} .
$$

The corresponding anomalous theoretical thresholds in units of the three-pion threshold $q_{\mathrm{th}}^{2}$ are

$$
\begin{gathered}
\frac{q_{\Lambda K^{-}}^{2} p}{q_{\mathrm{th}}^{2}}=\frac{4 M_{K}^{2}}{q_{\mathrm{th}}^{2}}-\frac{\left(M_{\Lambda}^{2}-M_{p}^{2}-M_{K}^{2}\right)^{2}}{M_{p}^{2} q_{\mathrm{th}}^{2}} \simeq 5.59, \\
\frac{q_{\Lambda K^{0} n}^{2}}{q_{\mathrm{th}}^{2}}=\frac{4 M_{K^{0}}^{2}}{q_{\mathrm{th}}^{2}}-\frac{\left(M_{\Lambda}^{2}-M_{n}^{2}-M_{K^{0}}^{2}\right)^{2}}{M_{n}^{2} q_{\mathrm{th}}^{2}} \simeq 5.69 .
\end{gathered}
$$

This means that the relative branch cuts originate well above the theoretical threshold and hence no modification of the dispersive procedure is needed because such anomalous branch cuts are included in the standard one. In more detail, the set of self-containing cuts, whose first elements are listed in the sequence of inclusions of Eq. (7), has to be updated, it becomes

$$
\begin{gathered}
\underbrace{\left(q_{\mathrm{th}}^{2}, \infty\right)}_{\pi^{+} \pi^{-} \pi^{0} \text { cut }} \supset \underbrace{\left(M_{\omega}^{2}, \infty\right)}_{\omega \text { cut }} \supset \underbrace{\left(\left(M_{\rho}+M_{\pi}\right)^{2}, \infty\right)}_{\rho \pi \text { cut }} \\
\supset \underbrace{\left(q_{\Lambda K^{-} p}^{2}, \infty\right)}_{\Lambda K^{-} p \text { anomalous cut }} \supset \underbrace{\left(q_{\Lambda K^{0} n}^{2}, \infty\right)}_{\Lambda K^{0} n \text { anomalous cut }} \supset \\
\supset \underbrace{\left(4 M_{K}^{2}, \infty\right)}_{K^{+} K^{-} \text {cut }} \supset \underbrace{\left(4 M_{K^{0}}^{2}, \infty\right)}_{K_{S}^{0} K_{L}^{0} \text { cut }} \supset \cdots .
\end{gathered}
$$

(d) Asymptotic behavior. The high- $\left|q^{2}\right|$ behavior of the FFs is obtained in the framework of the perturbative 
QCD that predicts the same power law for both FFs [16,17], namely

$$
G_{E, M}^{\Lambda}\left(q^{2}\right)=\mathcal{O}\left(\left(q^{2}\right)^{-2}\right), \quad q^{2} \rightarrow-\infty .
$$

In fact, at high $q^{2}$ the dominant amplitude of the spacelike process $\gamma^{*} B \rightarrow B$, where the virtual photon $\gamma^{*}$ is absorbed by the baryon $B$, is that containing two gluon propagators and hence it scales like $\left(q^{2}\right)^{-2}$. This is a consequence of the fact that, in order to maintain the baryon intact, the four-momentum transferred by the virtual photon has to be shared among the constituent quarks through multiple gluon exchanges and, at high $q^{2}$, the most probable reaction mechanism is that involving the minimum number of gluon exchanges that are needed for the complete fourmomentum distribution among the three constituent quarks of the baryon.

Moreover, the analyticity implies that the FFs have to have the same asymptotic behavior in spacelike and timelike regions, and hence the high- $\left(-q^{2}\right)$ trend described in Eq. (10) is valid also in the timelike limit $q^{2} \rightarrow \infty$. It is straightforward to infer that the ratio of FFs is asymptotically constant. This is the obvious consequence of the fact that the high- $\left|q^{2}\right|$ trend of both FFs is ruled by the same power law which is given in Eq. (10). Hence, by neglecting QCD corrections, the ratio scales like

$$
R\left(q^{2}\right)=\mathcal{O}(1), \quad\left|q^{2}\right| \rightarrow \infty .
$$

On the other hand, the inclusion of QCD corrections does affect the high- $\left|q^{2}\right|$ behavior of the ratio. However, there is no consensus on how to implement such corrections; different methods lead to different results for the asymptotic behavior of FFs and $R\left(q^{2}\right)$. Two examples are described in Appendix C.

Even though some QCD-correction schemes provide a logarithmically divergent trend for the ratio $R\left(q^{2}\right)$ as $\left|q^{2}\right| \rightarrow \infty$, DRs with a single subtraction (see Appendix A) still remain applicable, because of the convergence condition $R\left(q^{2}\right) / q^{2}=o\left(\ln \left(\left|q^{2}\right|\right)\right)$, as $\left|q^{2}\right| \rightarrow \infty$, is verified.

Finally, from the extension of the power-law behavior of Eq. (10) to the timelike limit $q^{2} \rightarrow \infty$, it follows that

$$
\lim _{q^{2} \rightarrow \infty} \frac{G_{E, M}^{\Lambda}\left(q^{2}\right)}{G_{E, M}^{\Lambda}\left(-q^{2}\right)}=1
$$

This means that not only FFs scale with the same power law but also that they have just the same timelike and spacelike limits. Thus their ratio, timelike over spacelike in Eq. (11), tends to unity. A further consequence is that, since the timelike FFs are complex functions with non-null imaginary parts, such imaginary parts have to vanish faster than the real ones in the limit $q^{2} \rightarrow \infty$.

\section{THE PARAMETRIZATION FOR $R\left(q^{2}\right)$ AND THE $\chi^{2}$ DEFINITION}

The imaginary part of the ratio is parametrized as a combination of Chebyshev polynomials of the first kind $T_{j}(x)$ [18], with $j \in \mathbb{N} \cup\{0\}$ and $x \in[-1,1]$. These polynomials can be defined through the trigonometric form

$$
T_{j}(\cos (\alpha))=\cos (j \alpha),
$$

with $\alpha \in[0,2 \pi]$. In this form, the values at the extremes $x=+1,-1$, corresponding to $\alpha=0, \pi$, are

$$
T_{j}(-1)=\cos (j \pi)=(-1)^{j}, \quad T_{j}(1)=\cos (0)=1 .
$$

We define the parametrization

$$
\begin{aligned}
\operatorname{Im}\left[R\left(q^{2}\right)\right] & \equiv Y\left(q^{2} ; \vec{C}, q_{\text {asy }}^{2}\right) \\
Y\left(q^{2} ; \vec{C}, q_{\text {asy }}^{2}\right) & = \begin{cases}\sum_{j=0}^{N} C_{j} T_{j}\left[x\left(q^{2}\right)\right] & q_{\text {th }}^{2}<q^{2}<q_{\text {asy }}^{2} \\
0 & q^{2} \geq q_{\text {asy }}^{2}\end{cases}
\end{aligned}
$$

where

$$
x\left(q^{2}\right)=2 \frac{q^{2}-q_{\mathrm{th}}^{2}}{q_{\text {asy }}^{2}-q_{\mathrm{th}}^{2}}-1 .
$$

It depends on the set of $N+2$ free parameters $\left\{\vec{C}=\left(C_{0}, C_{1}, \ldots, C_{N}\right), q_{\text {asy }}^{2}\right\}$, namely the $N+1$ coefficients of the Chebyshev polynomials representing the components of the vector $\vec{C} \in \mathbb{R}^{N+1}$, together with the asymptotic threshold $q_{\text {asy }}^{2} \in\left(q_{\text {phy }}^{2}, \infty\right)$.

The free parameters are determined by imposing theoretical and experimental constraints. While for the latter a standard $\chi^{2}$ minimization procedure is adopted, the former, which actually represents a set of conditions that have to be exactly fulfilled, are forced by means of two different methods. Those regarding directly the imaginary part, i.e., known values at some well-established $q^{2}$, are forced by fixing a subset of the Chebyshev coefficients. The other theoretical constraints which concern the real part, obtained from the imaginary part through the subtracted DR of Eq. (A6), are imposed by means of a $\chi^{2}$ minimization.

The theoretical constraints on the imaginary part, parametrized as given in Eq. (12), follow from the reality of the 
FF ratio at the theoretical and physical thresholds, as well as in the asymptotic region $\left(q_{\text {asy }}^{2}, \infty\right)$. They can be imposed by the following three conditions

$$
\begin{cases}R\left(q_{\mathrm{th}}^{2}\right) \in \mathbb{R} & \Rightarrow Y\left(q_{\mathrm{th}}^{2} ; \vec{C}, q_{\mathrm{asy}}^{2}\right)=0 \\ R\left(q_{\mathrm{phy}}^{2}\right) \in \mathbb{R} & \Rightarrow Y\left(q_{\mathrm{phy}}^{2} ; \vec{C}, q_{\text {asy }}^{2}\right)=0 \\ R\left(q^{2} \geq q_{\mathrm{asy}}^{2}\right) \in \mathbb{R} & \Rightarrow Y\left(q^{2} \geq q_{\text {asy }}^{2} ; \vec{C}, q_{\text {asy }}^{2}\right)=0\end{cases}
$$

These identities represent three equations of a linear system having, as unknowns, three arbitrary Chebyshev coefficients, e.g., $C_{0}, C_{1}$, and $C_{2}$, so that the number of degrees of freedom for the parametrization of Eq. (12) reduces from $N+2$ to $N-1$, i.e., $N-2$ Chebyshev coefficients together with the asymptotic threshold $q_{\mathrm{asy}}^{2}$.

The other theoretical constraints concern the value of the real part of the ratio. In particular, at the physical threshold $q_{\text {phy }}^{2}$, where the FFs are equal each other, the ratio is not only real but is equal to one and hence the condition is

$$
\operatorname{Re}\left[R\left(q_{\text {phy }}^{2}\right)\right]=\frac{q_{\text {phy }}^{2}}{\pi} \operatorname{Pr} \int_{q_{\text {th }}^{2}}^{q_{\text {asy }}^{2}} \frac{Y\left(s ; \vec{C}, q_{\text {asy }}^{2}\right)}{s\left(s-q_{\text {phy }}^{2}\right)} d s=1 .
$$

The possibility of forcing the convergence to the unity, as $q^{2} \rightarrow \infty$ of the modulus of real part $\operatorname{Re}\left[R\left(q^{2}\right)\right]$ is also considered in accordance with the perturbative QCD prediction. It is assumed that such a limit value is attained at the asymptotic threshold $q_{\text {asy }}^{2}$, and constantly maintained for $q^{2} \geq q_{\text {asy. }}^{2}$. This eventuality is accounted for by requiring

$$
\left|\operatorname{Re}\left[R\left(q^{2}\right)\right]\right|=\frac{q^{2}}{\pi}\left|\operatorname{Pr} \int_{q_{\text {th }}^{2}}^{q_{\text {asy }}^{2}} \frac{Y\left(s ; \vec{C}, q_{\text {asy }}^{2}\right)}{s\left(s-q^{2}\right)} d s\right|=1,
$$

for $q^{2} \geq q_{\text {asy }}^{2}$.

Concerning the experimental conditions, as already discussed, the only available data are:

(a) on the modulus of the ratio, $\left|R\left(q^{2}\right)\right|$ : two points from BESIII and one point from $B A B A R$, the corresponding set is $\left\{q_{j}^{2},\left|R_{j}\right|, \delta\left|R_{j}\right|\right\}_{j=1}^{M}$, with $M=3$;

(b) on the sinus of the phase $\phi\left(q^{2}\right)=\arg \left(R\left(q^{2}\right)\right)$ : one point from BESIII and one from BABAR, the set is $\left\{q_{k}^{2}, \sin \left(\phi_{k}\right), \delta \sin \left(\phi_{k}\right)\right\}_{k=1}^{P}$, with $P=2$.

The $\chi^{2}$, depending on the set of $N-1$ free parameters $\left\{C_{3}, C_{4}, \ldots, C_{N}, q_{\text {asy }}^{2}\right\}$ [the first three Chebyshev coefficients have been fixed by the linear system of Eq. (14)], and accounting for all these conditions is defined as

$$
\begin{aligned}
\chi^{2}\left(\vec{C}, q_{\text {asy }}^{2}\right)= & \chi_{|R|}^{2}+\chi_{\phi}^{2} \\
& +\tau_{\text {phy }} \chi_{\text {phy }}^{2}+\tau_{\text {asy }} \chi_{\text {asy }}^{2}+\tau_{\text {curv }} \chi_{\text {curv }}^{2} .
\end{aligned}
$$

The first two contributions imposing the experimental constraints, are

$$
\begin{aligned}
\chi_{|R|}^{2} & =\sum_{j=1}^{M}\left(\frac{\sqrt{X\left(q_{j}^{2}\right)^{2}+Y\left(q_{j}^{2}\right)^{2}}-\left|R_{j}\right|}{\delta\left|R_{j}\right|}\right)^{2}, \\
\chi_{\phi}^{2} & =\sum_{k=1}^{P}\left(\frac{\sin \left(\arctan \left(Y\left(q_{k}^{2}\right) / X\left(q_{k}^{2}\right)\right)\right)-\sin \left(\phi_{k}\right)}{\delta \sin \left(\phi_{k}\right)}\right)^{2},
\end{aligned}
$$

where $X\left(q^{2}\right)$ is the real part obtained through the DR

$$
X\left(q^{2}\right) \equiv \operatorname{Re}\left(R\left(q^{2}\right)\right)=\frac{q^{2}}{\pi} \operatorname{Pr} \int_{q_{\mathrm{th}}^{2}}^{q_{\mathrm{asy}}^{2}} \frac{Y(s)}{s\left(s-q^{2}\right)} d s .
$$

The third and fourth contributions impose, with adjustable weights $\tau_{\text {phy }}$ and $\tau_{\text {asy }}$, the constraints on the real part of the ratio at the physical and asymptotic thresholds, $q_{\text {phy }}^{2}$ and $q_{\text {asy }}^{2}$, i.e.,

$$
\chi_{\text {phy }}^{2}=\left(1-X\left(q_{\text {phy }}^{2}\right)\right)^{2}, \quad \chi_{\text {asy }}^{2}=\left(1-X\left(q_{\text {asy }}^{2}\right)^{2}\right)^{2} .
$$

The latter is written for the squared value because the perturbative QCD prediction concerns the modulus of the real part and hence both values $X\left(q_{\text {asy }}^{2}\right)= \pm 1$ have to be considered.

The last contribution $\tau_{\text {curv }} \chi_{\text {curv }}^{2}$ is introduced to stabilize the solution of a so-called ill-posed problem, usually represented by a Fredholm integral equation [19].

For instance, the inhomogeneous Fredholm integral equation of the second kind

$$
u(x)=\int_{c}^{d} K\left(x, x^{\prime}\right) u\left(x^{\prime}\right) d x^{\prime}+\phi(x), \quad \begin{aligned}
x & \in[a, b] \\
x^{\prime} & \in[c, d]
\end{aligned},
$$

where $\phi(x), u(x)$, and $K\left(x, x^{\prime}\right)$ are the input function, the unknown function and the kernel, can be solved by using the Tikhonov regularization procedure [19]. Such a procedure consists of minimizing the functional

$F[u]=\int_{a}^{b}\left|u(x)-\phi(x)-\int_{c}^{d} K\left(x, x^{\prime}\right) u\left(x^{\prime}\right) d x^{\prime}\right|^{2} d x+\tau C[u]$,

where the interval $[a, b] \subset \mathbb{R}$ is the domain of the input function $\phi(x)$, the real non-negative number $\tau$ is the regularization parameter, and the functional $C[u]$ represents the total curvature of the solution $u(x)$ for $x \in[c, d]$, i.e.,

$$
C[u]=\int_{c}^{d}\left|\frac{d^{2} u}{d x^{2}}\right|^{2} d x .
$$

It follows that the total curvature of the solution obtained by minimizing the functional $F[u]$ with a certain value of $\tau$, is large for a small value of $\tau$, and small for a large value of $\tau$. 
The extreme cases are those with $\tau \rightarrow \infty$, giving constant $u(x)$, and $\tau=0$ resulting, instead, in a highly-oscillating solution.

In our case the functional to be minimized is the $\chi^{2}$ of Eq. (15) and the unknown function is $Y\left(q^{2}\right)$, the imaginary part of the ratio defined in Eq. (12). The real part $X\left(q^{2}\right)$ is given by the DR integral of Eq. (16), and it is just this quantity that gives the "integral" character to the minimization problem. As a consequence, the contribution $\chi_{\text {curv }}^{2}$ to the total $\chi^{2}$ has the form

$$
\chi_{\text {curv }}^{2}=\int_{q_{\mathrm{th}}^{2}}^{q_{\mathrm{asy}}^{2}}\left|\frac{d^{2} Y(s)}{d s^{2}}\right|^{2} d s,
$$

and it represents the total curvature of $Y\left(q^{2}\right)$ in the $q^{2}$ interval $\left[q_{\mathrm{th}}^{2}, q_{\mathrm{asy}}^{2}\right]$ where it is not constant.

The values of the weights $\tau_{\text {phy }}$ and $\tau_{\text {asy }}$ have been chosen in order to make the fulfillment exact. In particular, it happens that starting from a threshold value, $\tau_{\text {phy,asy }}=\tau_{\text {phy,asy }}^{0}$, the corresponding $\chi^{2}$ contribution turns out to be negligible with respect to the others so that the minimization procedure becomes independent of their values. On the basis of these considerations the minimization procedure has been carried out by setting $\tau_{\text {phy }}=\tau_{\text {phy }}^{0}$ and $\tau_{\text {asy }}=\tau_{\text {asy }}^{0}$. Additionally, the value of the regularization parameter $\tau_{\text {curv }}$, which through the $\chi^{2}$ minimization procedure allows us to control the total curvature of $Y\left(q^{2}\right)$ in the $q^{2}$ interval $\left[q_{\mathrm{th}}^{2}, q_{\mathrm{asy}}^{2}\right]$, has to be determined by means of a more refined technique.

The parametrization in terms of the combination of the first $N+1$ Chebyshev's polynomials, and hence the degree $N$ of the resulting polynomial in $q^{2}$ given in Eq. (12), represents by itself a solution with a controlled curvature. No more than $N$ extrema, (maxima plus minima, i.e., zeros of the first derivative of the polynomial) can be present in any solution. This means that the number of oscillations is limited. On the other hand, the constraint represented by the DRs integral limits the amplitude of such oscillations. It follows that the combined effect of these two features of the parametrization does not allow largely unstable solutions. Nevertheless, the ill-posedness nature of the inverse problem has to be faced because when the regularization parameter $\tau_{\text {curv }}$ is set to zero the solution always has the maximum curvature, i.e., the maximum number of oscillations allowed by the degree of the polynomial.

In the light of that, the degree $N$ and the regularization parameter $\tau_{\text {curv }}$ are mutually dependent. The selection of their values has to be made by suitably balancing two opposite tendencies: the increase of the total curvature as the polynomial degree increases; the progressive suppression of the oscillations, and hence the reduction of the curvature, achieved by increasing the value of the regularization parameter $\tau_{\text {curv }}$.

In addition, to select the final values of $N$ and $\tau_{\text {curv }}$, we also consider the role played by the free parameter $q_{\text {asy }}^{2}$,

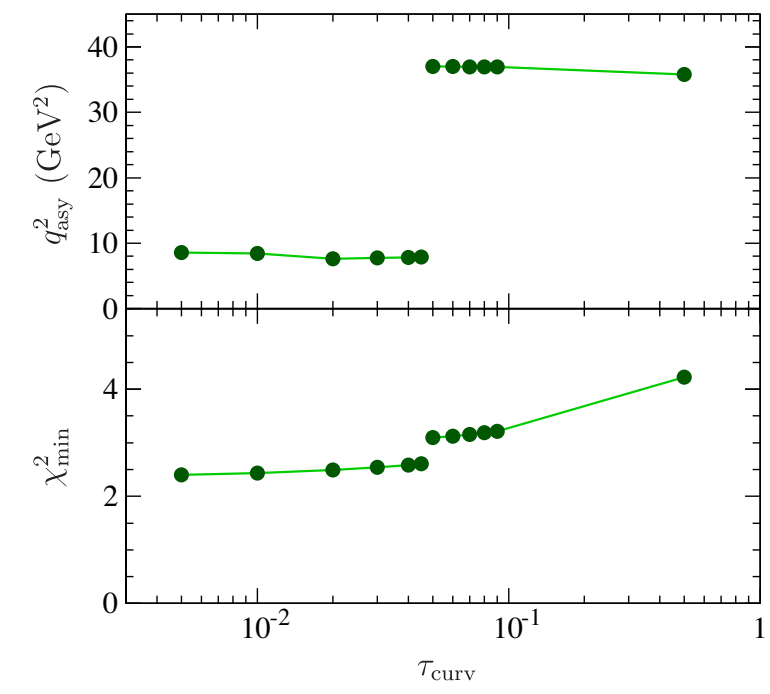

FIG. 5. Best values of the parameter $q_{\text {asy }}^{2}$ and minima of the total $\chi^{2}$ defined in Eq. (15) as a function of the regularization parameter $\tau_{\text {curv }}$, with $N=5$.

representing the $q^{2}$ threshold from which the asymptotic behavior is assumed. In particular, solutions with larger values of such a free parameter are evidently privileged.

Figure 5 shows the best values of $q_{\text {asy }}^{2}$ and the corresponding $\chi^{2}$ minima with $N=5$ as a function of $\tau_{\text {curv }}$, at which the minimization is performed. At $\tau_{\text {curv }}=0.05$ there is a kind of phase transition where the best value for the asymptotic threshold moves from $q_{\text {asy }}^{2} \simeq 8 \mathrm{GeV}^{2}$ to a larger value $q_{\text {asy }}^{2} \simeq 37 \mathrm{GeV}^{2}$, which is more reliable according to its own definition. As a consequence of such a widening of the $q^{2}$ interval covered by the polynomial parametrization, $\left[q_{\mathrm{th}}^{2}, q_{\mathrm{asy}}^{2}\right]$, there is a limited but acceptable $(\leq 20 \%)$ increase of the minimum $\chi^{2}$. The final values for the regularization parameter and the highest-polynomial degree have then been chosen as

$$
\tau_{\text {curv }}=0.05, \quad N=5 .
$$

In this case, as discussed in detail in Appendix B, having five data points and four free parameters, there is only one degree of freedom. It follows that the $\chi^{2}$ defined in Eq. (15) and shown in the lower panel of Fig. 5, referring to a single degree of freedom, is already normalized.

\section{RESULTS AND DISCUSSION}

The very few available data and their large uncertainties represent an obstacle to the complete determination of the FF ratio as a complex function of $q^{2}$ in the timelike region, above the theoretical threshold $q_{\mathrm{th}}^{2}$, and, as a real function of $q^{2}$ in the remaining kinematical range. Since, as already stated, at these energies the FFs and hence their ratio are real, the relative phase assumes values that are integer 
multiples of $\pi$ radiants. Such an eventuality is accounted for by defining the corresponding integers $N_{\text {th }}$ and $N_{\text {asy }}$ as

$$
N_{\mathrm{th}}=\frac{1}{\pi} \arg \left(\frac{G_{E}^{\Lambda}\left(q_{\mathrm{th}}^{2}\right)}{G_{M}^{\Lambda}\left(q_{\mathrm{th}}^{2}\right)}\right), \quad N_{\mathrm{asy}}=\frac{1}{\pi} \arg \left(\frac{G_{E}^{\Lambda}\left(q_{\text {asy }}^{2}\right)}{G_{M}^{\Lambda}\left(q_{\mathrm{asy}}^{2}\right)}\right) .
$$

As already widely discussed and exhaustively argued in the previous sections, the theoretical constraints based on the first principles of quantum field theory and analyticity of the FFs offer a unique possibility to achieve educated guesses on the phase determination of the imaginary and real part and hence the modulus and phase of the ratio $G_{E}^{\Lambda} / G_{M}^{\Lambda}$ by relating through the DR of Eq. (16), It is important to reiterate that this phase determination embodies crucial physical information which is not experimentally accessible since the experiments measure only the sinus of the phase.

Unfortunately, the only available five data points with their large uncertainties do not allow us to obtain unique values for $N_{\text {th }}$ and $N_{\text {asy }}$. Nevertheless, the number of possible solutions that, in principle are infinite, is drastically reduced to the few possibilities reported in Table I. Furthermore, the Monte Carlo procedure, built to perform a statistical analysis of our results, described in Appendix B, also gives the probability of the occurrence of each pair $\left(N_{\text {th }}, N_{\text {asy }}\right)$. These probabilities are reported as percentages and visual strips in Table I and as a three-dimensional histogram on a $N_{\text {th }} N_{\text {asy }}$-grid in Fig. 6.

We classify the different descriptions of the two observables, phase and modulus of the FF ratio $G_{E}^{\Lambda} / G_{M}^{\Lambda}$ in terms of the pairs $\left(N_{\text {th }}, N_{\text {asy }}\right)$, obtained through our procedure which is based on DRs and experimental data.

By considering the cases having a probability of occurrence larger than $0.5 \%$ as acceptable, only six pairs $\left(N_{\text {th }}, N_{\text {asy }}\right)$ are phenomenologically compatible with the data. Once again, the fact that so few cases are selected despite the lack of experimental knowledge of the pivotal observables, namely the modulus and the phase of the ratio $G_{E}^{\Lambda} / G_{M}^{\Lambda}$, proves the strength and the effectiveness of the theoretical frame of the procedure.

TABLE I. Numerical and visual percentage of cases corresponding to the values of the phase, in units of $\pi$ radiants, at the theoretical threshold $q_{\mathrm{th}}^{2}$ and at $q_{\text {asy }}^{2}$.

\begin{tabular}{lcrll}
\hline \hline$N_{\text {th }}$ & $N_{\text {asy }}$ & \multicolumn{1}{c}{$\%$} & Visual percentage \\
\hline-1 & 0 & 4.0 & & \\
-1 & 1 & 16.0 & \\
-1 & 2 & 50.5 & & \\
-1 & 3 & 0.7 & & \\
0 & 1 & 0.3 & 1 & \\
0 & 3 & 26.8 & \\
1 & 2 & 0.1 & & \\
1 & 3 & 1.6 & \\
\hline \hline
\end{tabular}

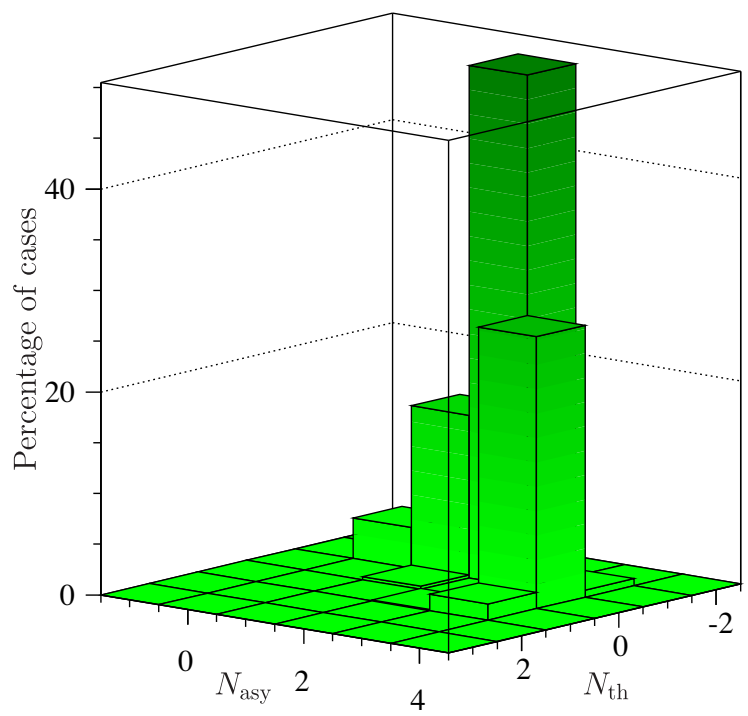

FIG. 6. Percentage of cases characterized by the values of the phases, in units of $\pi$ radiants, at the theoretical threshold $q_{\text {th }}^{2}$ and at $q_{\text {asy }}^{2}$.

The results obtained for the modulus and the phase of $G_{E}^{\Lambda} / G_{M}^{\Lambda}$ corresponding to the six cases $\left(N_{\text {th }}, N_{\text {asy }}\right)=$ $(-1,0),(-1,1),(-1,2),(-1,3),(0,3),(1,3)$ are shown in Fig. 7, from the upper-left to the lower-right panel, respectively.

At the theoretical threshold the phase of the ratio can assume only three possible values: 0 or $\pm \pi$, i.e., $N_{\text {th }}=0$ or $N_{\text {th }}= \pm 1$. They depend on the sign of the real values of the FFs $G_{E}^{\Lambda}$ and $G_{M}^{\Lambda}$ just below the theoretical threshold $q_{\mathrm{th}}^{2}$, above which they acquire a nonvanishing imaginary part. In particular:

(a) $N_{\text {th }}=0$, if the FFs $G_{E}^{\Lambda}$ and $G_{M}^{\Lambda}$ at $q^{2} \rightarrow q_{\mathrm{th}}^{2-}$ have the same sign, i.e.,

$$
\left\{\begin{array}{l}
G_{E}^{\Lambda}\left(q^{2}\right) \underset{q^{2} \rightarrow q_{\mathrm{th}}^{2}}{\longrightarrow}+(-)\left|G_{E}^{\Lambda}\left(q_{\mathrm{th}}^{2}\right)\right|=\left|G_{E}^{\Lambda}\left(q_{\mathrm{th}}^{2}\right)\right| e^{0(i \pi)} \\
G_{M}^{\Lambda}\left(q^{2}\right) \underset{q^{2} \rightarrow q_{\mathrm{th}}^{2}}{\longrightarrow}+(-)\left|G_{M}^{\Lambda}\left(q_{\mathrm{th}}^{2}\right)\right|=\left|G_{M}^{\Lambda}\left(q_{\mathrm{th}}^{2}\right)\right| e^{0(i \pi)}
\end{array} ;\right.
$$

(b) $N_{\text {th }}=+1$, if at $q^{2} \rightarrow q_{\text {th }}^{2-}$ the electric FF is negative and the magnetic is positive,

$$
\left\{\begin{array}{l}
G_{E}^{\Lambda}\left(q^{2}\right) \underset{q^{2} \rightarrow q_{\mathrm{th}}^{2}-}{\longrightarrow}-\left|G_{E}^{\Lambda}\left(q_{\mathrm{th}}^{2}\right)\right|=\left|G_{E}^{\Lambda}\left(q_{\mathrm{th}}^{2}\right)\right| e^{i \pi} \\
G_{M}^{\Lambda}\left(q^{2}\right) \underset{q^{2} \rightarrow q_{\mathrm{th}}^{2}}{\longrightarrow}\left|G_{M}^{\Lambda}\left(q_{\mathrm{th}}^{2}\right)\right|=\left|G_{M}^{\Lambda}\left(q_{\mathrm{th}}^{2}\right)\right| e^{0}
\end{array} ;\right.
$$

(c) $N_{\mathrm{th}}=-1$, if at $q^{2} \rightarrow q_{\mathrm{th}}^{2-}$ the electric FF is positive and the magnetic is negative, 

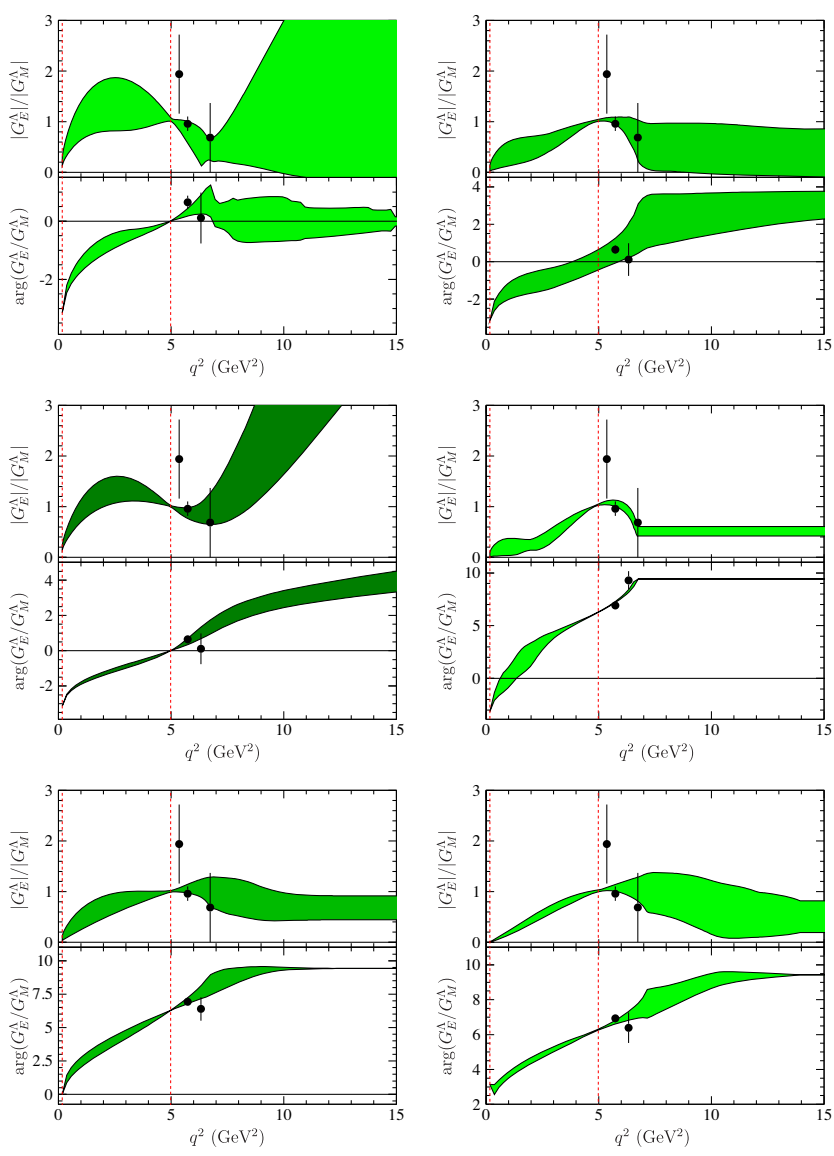

FIG. 7. Modulus (upper panels) and phase (lower panels) of the FF ratio with: $\left(N_{\text {th }}, N_{\text {asy }}\right)=(-1,0)$ in the upper-left position, $\left(N_{\text {th }}, N_{\text {asy }}\right)=(-1,1)$ in the upper-right position, $\left(N_{\text {th }}, N_{\text {asy }}\right)=$ $(-1,2)$ in the middle-left position, $\left(N_{\text {th }}, N_{\text {asy }}\right)=(-1,3)$ in middle-right position, $\left(N_{\text {th }}, N_{\text {asy }}\right)=(0,3)$ in the lower-left position, $\left(N_{\text {th }}, N_{\text {asy }}\right)=(1,3)$ in the lower-right position. The color intensity of the error bands is proportional to the occurrence probability of the corresponding pair $\left(N_{\text {th }}, N_{\text {asy }}\right)$; a darker color indicates an higher probability. The red dashed lines indicate the theoretical and the physical thresholds.

$$
\left\{\begin{array}{l}
G_{E}^{\Lambda}\left(q^{2}\right) \underset{q^{2} \rightarrow q_{\mathrm{th}}^{2}}{\longrightarrow}+\left|G_{E}^{\Lambda}\left(q_{\mathrm{th}}^{2}\right)\right|=\left|G_{E}^{\Lambda}\left(q_{\mathrm{th}}^{2}\right)\right| e^{0} \\
G_{M}^{\Lambda}\left(q^{2}\right) \underset{q^{2} \rightarrow q_{\mathrm{th}}^{2-}}{\longrightarrow}-\left|G_{M}^{\Lambda}\left(q_{\mathrm{th}}^{2}\right)\right|=\left|G_{M}^{\Lambda}\left(q_{\mathrm{th}}^{2}\right)\right| e^{i \pi} .
\end{array}\right.
$$

Concerning the asymptotic behavior, we assume that from $q^{2}=q_{\text {asy }}^{2}$ up to infinity the FF ratio stays real, and hence the phase in this $q^{2}$ region is constant and equal to the integer multiple of $\pi$ radians $N_{\text {asy }} \pi$. Moreover, assuming that the magnetic FF has no zeros in the $q^{2}$-complex plane with the branch cut $\left(q_{\mathrm{th}}^{2}, \infty\right)$, so that the ratio, having no poles, is analytic in the same domain, we can invoke the Levinson's theorem [Eq. (5)] to infer that $N_{\text {asy }}$ must be greater than $N_{\text {th }}$. In the peculiar case of the neutral $\Lambda$ baryons, the normalization at the origin of the electric FF to the total charge, i.e., $G_{E}^{\Lambda}(0)=0$ implies

$$
N_{\text {asy }} \geq N_{\text {th }}+1 \text {. }
$$

The unity in the right-hand side accounts for the zero at $q^{2}=0$ of the ratio $G_{E}^{\Lambda} / G_{M}^{\Lambda}$, which is the consequence of the zero of $G_{E}^{\Lambda}$ and of the nonvanishing value of $G_{M}^{\Lambda}(0)=\mu_{\Lambda} \neq 0$, as is shown in Eq. (6).

Figure 7 is a grid of six double graphs which show the modulus and the phase of the ratio in the upper and lower panels, respectively, for each of the six pairs of $\left(N_{\text {th }}, N_{\text {asy }}\right)$ having an occurrence probability larger than $0.5 \%$ (see Table I). The error bands have been obtained by the standard statistical analysis of the results obtained with the Monte Carlo procedure described in Appendix B. The best values of the seven parameters $\left\{C_{0}, C_{1}, C_{2}, C_{3}, C_{4}, C_{5}, q_{\text {asy }}^{2}\right\}$, of which only four are free, namely three Chebyshev's coefficients and $q_{\text {asy }}^{2}$, resulting from the $\chi^{2}$ minimization are reported in Table II of the same appendix. It is interesting to notice how the procedure works in establishing the determination of the phase. Different determinations are assigned to the phase values extracted from the same data on its sinus (insensitive to the determination) once the connection with the modulus is established through DRs and after a set of crucial theoretical constraints is imposed. In particular, the phase values extracted from the most precise data on the sinus, namely that provided by the BESIII Collaboration [7], shown as a dark green square in the right panel of Fig. 2, has been used as the reference point to which the fundamental determination is assigned, namely $2 k \pi<\arg \left(G_{E}^{\Lambda} / G_{M}^{\Lambda}\right)<(2 k+1 / 2) \pi$, with $0<\sin \left(\arg \left(G_{E}^{\Lambda} / G_{M}^{\Lambda}\right)\right)<1$, and with the integer $k \in$ $\mathbb{N} \cup\{0\}$ obtained by the dispersive analysis.

The determination of the BABAR point has been fixed as a consequence of this choice and on the light of the result of the dispersive analysis, which selected one of the two possibilities, either $2 k \pi<\arg \left(G_{E}^{\Lambda} / G_{M}^{\Lambda}\right)<(2 k+1 / 2) \pi$, as for the BESIII point, or $(2 k+1 / 2) \pi<\arg \left(G_{E}^{\Lambda} / G_{M}^{\Lambda}\right)<(2 k+1) \pi$. As can be seen in the lower panel of the middle-right picture of Fig. 7, only in the case with $\left(N_{\text {th }}, N_{\text {asy }}\right)=(-1,3)$, where $k=1$, are the two fundamental determinations different. In fact, the BESIII phase lies in $2 \pi<\arg \left(G_{E}^{\Lambda} / G_{M}^{\Lambda}\right)<5 \pi / 2$, while the $B A B A R$ value lies in $5 \pi / 2<\arg \left(G_{E}^{\Lambda} / G_{M}^{\Lambda}\right)<3 \pi$. In more detail, the value of $k$ is zero in the first three cases, with $\left(N_{\text {th }}, N_{\text {asy }}\right)=(-1,0),(-1,1),(-1,2)$, while it is $k=$ 1 in the last three cases: $\left(N_{\text {th }}, N_{\text {asy }}\right)=(-1,3),(0,3),(1,3)$.

\section{A. The modulus of $G_{E}^{\Lambda} / G_{M}^{\Lambda}$}

The modulus of the FF ratio represents the best-established observable of this analysis since it is not affected by any ambiguity of definition as does the phase. Nevertheless, the lack of data, makes the predictions quite uncertain especially for the asymptotic behavior, which, in the majority of the cases concerns a $q^{2}$-region lying well above the higher available data point. 
TABLE II. Chebyshev's parameters and $q_{\text {asy }}^{2}$ obtained with $N=5$ in the six cases under consideration. There are $N-1=4$ free parameters, i.e., the last three coefficients: $C_{3}, C_{4}$ and $C_{5}$, and $q_{\text {asy }}^{2}$. The values of the first three Chebyshev's coefficients, $C_{0}, C_{1}, C_{2}$, are obtained as functions of the others by imposing the constraints of the three nodes for the imaginary part of the ratio at $q_{\mathrm{th}}^{2}, q_{\mathrm{phy}}^{2}$, and $q_{\mathrm{asy}}^{2}$ [see Eq. (14)].

\begin{tabular}{|c|c|c|c|c|c|c|c|}
\hline$\left(N_{\text {th }}, N_{\text {asy }}\right)$ & $C_{0}\left(C_{3}, C_{4}, C_{5}\right)$ & $C_{1}\left(C_{3}, C_{4}, C_{5}\right)$ & $C_{2}\left(C_{3}, C_{4}, C_{5}\right)$ & $C_{3}$ & $C_{4}$ & $C_{5}$ & $q_{\text {asy }}^{2}\left(\mathrm{GeV}^{2}\right)$ \\
\hline$(-1,0)$ & $-0.20 \pm 0.21$ & $0.33 \pm 0.22$ & $0.06 \pm 0.15$ & $-0.17 \pm 0.26$ & $0.14 \pm 0.20$ & $-0.17 \pm 0.12$ & $9.8 \pm 3.7$ \\
\hline$(-1,1)$ & $-0.18 \pm 0.68$ & $0.26 \pm 0.42$ & $0.21 \pm 0.48$ & $0.01 \pm 0.69$ & $-0.04 \pm 0.32$ & $-0.27 \pm 0.31$ & $10.9 \pm 5.7$ \\
\hline$(-1,2)$ & $-4.73 \pm 3.47$ & $-3.95 \pm 3.06$ & $3.91 \pm 3.38$ & $5.58 \pm 4.03$ & $0.82 \pm 0.16$ & $-1.62 \pm 0.97$ & $32.3 \pm 8.9$ \\
\hline$(-1,3)$ & $0.06 \pm 0.03$ & $0.31 \pm 0.05$ & $0.29 \pm 0.07$ & $0.01 \pm 0.05$ & $-0.36 \pm 0.06$ & $-0.32 \pm 0.08$ & $6.7 \pm 0.1$ \\
\hline$(0,3)$ & $0.15 \pm 0.10$ & $0.28 \pm 0.10$ & $0.28 \pm 0.20$ & $-0.34 \pm 0.25$ & $-0.43 \pm 0.16$ & $0.06 \pm 0.17$ & $8.1 \pm 1.3$ \\
\hline$(1,3)$ & $0.16 \pm 0.15$ & $0.40 \pm 0.08$ & $-0.04 \pm 0.24$ & $-0.51 \pm 0.16$ & $-0.12 \pm 0.11$ & $0.11 \pm 0.10$ & $10.1 \pm 1.8$ \\
\hline
\end{tabular}

In particular, the procedure gives as outcomes for each pairs $\left(N_{\text {th }}, N_{\text {asy }}\right)$ the asymptotic threshold $\left(q_{\text {asy }}^{2} \pm \delta q_{\text {asy }}^{2}\right)$, as well as the corresponding values of the modulus of the ratio $\left(R_{\text {asy }} \pm \delta R_{\text {asy }}\right)$, where $R_{\text {asy }}$ is defined as $R_{\text {asy }} \equiv\left|R\left(q_{\text {asy }}^{2}\right)\right|$. The obtained values of these asymptotic parameters are reported in Fig. 8 as ellipses centered at $\left(q_{\text {asy }}^{2}, R_{\text {asy }}\right)$ and having as the horizontal and vertical semiaxes the uncertainties $\delta q_{\text {asy }}^{2}$ and $\delta R_{\text {asy }}$, respectively.

From Fig. 8 it is clear that the large errors do not allow us to make any precise prediction on the asymptotic value of the ratio. It is interesting to notice how the convergence $\left|G_{E}^{\Lambda}\left(q^{2}\right)\right| \rightarrow\left|G_{M}^{\Lambda}\left(q^{2}\right)\right|$ as $q^{2} \rightarrow \infty$ is compatible with any case, apart from the one with $\left(N_{\text {th }}, N_{\text {asy }}\right)=(-1,2)$. Such a

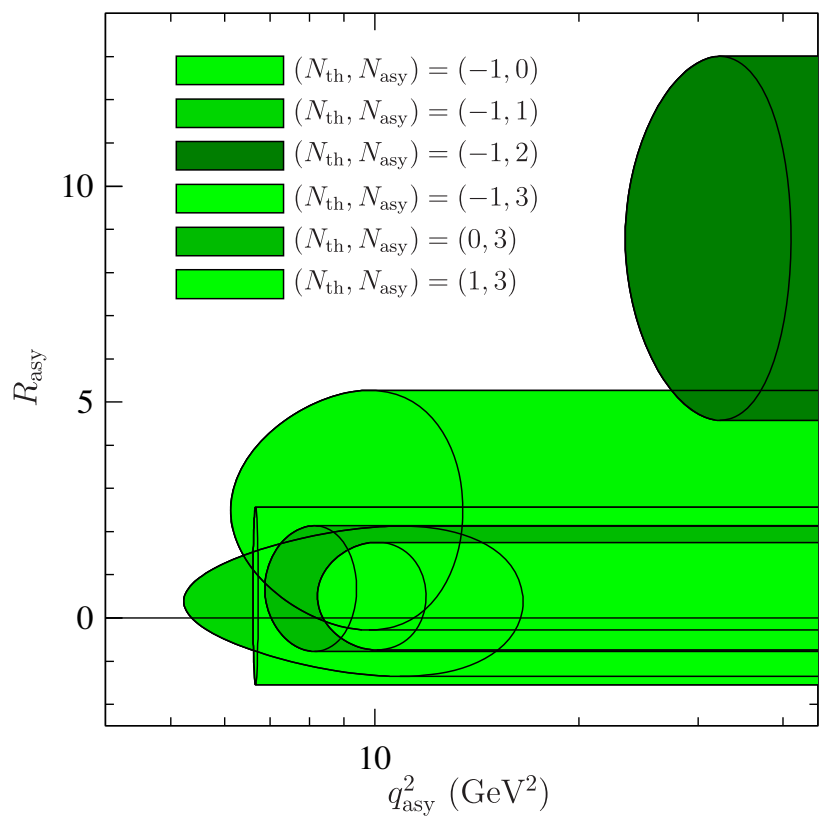

FIG. 8. Asymptotic behaviors for each case $\left(N_{\text {th }}, N_{\text {asy }}\right)$. The ellipses (deformed by the logarithmic scale) are centered at $\left(q_{\text {asy }}^{2}, R_{\text {asy }}\right)$, with $R_{\text {asy }} \equiv\left|R\left(q_{\text {asy }}^{2}\right)\right|$, and have the errors $\delta q_{\text {asy }}^{2}$ and $\delta R_{\text {asy }}$ as the horizontal and vertical semiaxes, respectively. The right-hand side tails represent the error bands of the asymptotic constant values. case is described by the darker green band, which corresponds to the most probable one (see Fig. 6 and Table I).

This also means that the knowledge of the modulus of the ratio at higher timelike $q^{2}$ would play a key role in disentangling the phase determination and hence in giving a hint on the pair $\left(N_{\text {th }}, N_{\text {asy }}\right)$ that describes the phase evolution with $q^{2}$, only in the case of $R_{\text {asy }}>6$, implying $\left(N_{\text {th }}, N_{\text {asy }}\right)=(-1,2)$.

On the other hand, a set of precise data on both the modulus of the ratio and its phase, preferably covering quite a wide range of $q^{2}$, would be certainly crucial in further reducing the possible cases, exactly as those already available have done in selecting from all possible pairs the only six that we have considered.

\section{B. The phase of $G_{E}^{\Lambda} / G_{M}^{\Lambda}$}

As stated above, experiments measure the sinus of the phase $\arg \left(G_{E}^{\Lambda} / G_{M}^{\Lambda}\right)$, not giving a direct determination of the phase. This is not a purely mathematical issue as it encodes precise information about the spacelike behavior of the analytic function under consideration, the ratio $G_{E}^{\Lambda} / G_{M}^{\Lambda}$ in our case.

It is interesting to notice that, even though a direct knowledge of the phase determination is prevented by the periodicity of the sinus function, it turns out to be somehow accessible by looking at the $q^{2}$-trend, i.e., the evolution of the sinus function itself as $q^{2}$ increases from the theoretical threshold $q_{\mathrm{th}}^{2}$ on. Indeed, having determined the starting value at $q_{\mathrm{th}}^{2}$ by means of the dispersive analysis, as one of the three possibilities: $\arg \left(G_{E}^{\Lambda}\left(q_{\mathrm{th}}^{2}\right) / G_{M}^{\Lambda}\left(q_{\mathrm{th}}^{2}\right)\right)=-\pi, 0, \pi$, i.e., $N_{\text {th }}=-1,0,1$, the determination at a certain $q^{2}$ is given by the number of oscillations that the sinus function has undertaken from $q_{\mathrm{th}}^{2}$ up to that value of $q^{2}$. Figure 9 shows just an example with $\left(N_{\text {th }}, N_{\text {asy }}\right)=(0,4)$, where a a given phase $\phi\left(q^{2}\right)$, upper panel, increases with $q^{2}$, starting from zero at the theoretical threshold and passing through $2 \pi$ radians at the physical threshold, where the ratio becomes again real and finally reaching the asymptotic regime at $q_{\text {asy }}^{2}$, at which point the ratio goes back to being real. From an experimental point of view the data on the sinus of such a phase should follow the oscillating behavior 


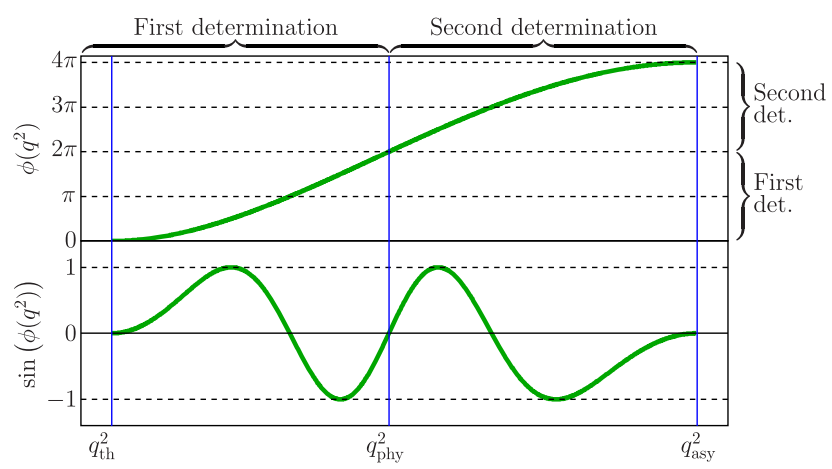

FIG. 9. The sinus of the phase $\phi\left(q^{2}\right)$, lower panel, undertakes two complete oscillations, having nodes at each of the three thresholds: $q_{\mathrm{th}}^{2}, q_{\mathrm{phy}}^{2}$, and $q_{\mathrm{asy}}^{2}$. In correspondence of such behavior of its sinus, the phase, upper panel, increases continuously, spanning the first and the second determination. It starts from zero at $q_{\mathrm{th}}^{2}$, reaching $2 \pi$ radians at $q_{\mathrm{phy}}^{2}$, and $4 \pi$ radians at the asymptotic threshold $q_{\text {asy }}^{2}$.

shown in the lower panel of Fig. 9, i.e., it should describe a complete period, reaching the maximum value +1 and the minimum -1 . This behavior, once the phase value at $q_{\text {phy }}^{2}$ is determined by the dispersive analysis, would unambiguously reveal the determination of the phase and hence the resulting spacelike features of the ratio. The message is that a measure covering a $q^{2}$ range as wide as possible would allow getting information about the phase with its own physical determination. The panels of Fig. 10 represent the results for the sinus of the phase $\arg \left(G_{E}^{\Lambda} / G_{M}^{\Lambda}\right)$ obtained in the six cases under study, namely those with $\left(N_{\text {th }}, N_{\text {asy }}\right)=$ $(-1,0),(-1,1),(-1,2),(-1,3),(0,3),(1,3)$. Following the scheme of the previous pictures, the level of darkness of the green areas describing the error bands, is proportional to the occurrence probability of the corresponding case. Unfortunately, we notice that in the results characterized by large values of $\Delta N=N_{\text {asy }}-N_{\text {th }}$, most of the oscillations of the sinus of the phase lie below the physical threshold and hence are not experimentally observable. Nevertheless, a better knowledge of this quantity, that is, more precise data and covering a larger energy region would be a pivotal step forward in the understanding of dynamics underlying the $\gamma \Lambda \bar{\Lambda}$ interaction.

\section{The $\Lambda$ charge radius}

The complete knowledge of the FF ratio $R\left(q^{2}\right)$, as an analytic complex function of $q^{2}$, defined in the whole $q^{2}$ complex plane, allows us to infer dynamical and also static features of the $\Lambda$ baryons.

One example is the so-called charge root-mean-square radius, $\left\langle r_{E}\right\rangle$, i.e., the square root of the mean square radius of the spatial charge distribution. In general, for an extended particle as a baryon, which have an electric FF $G_{E}\left(q^{2}\right)$, such a quantity is defined by
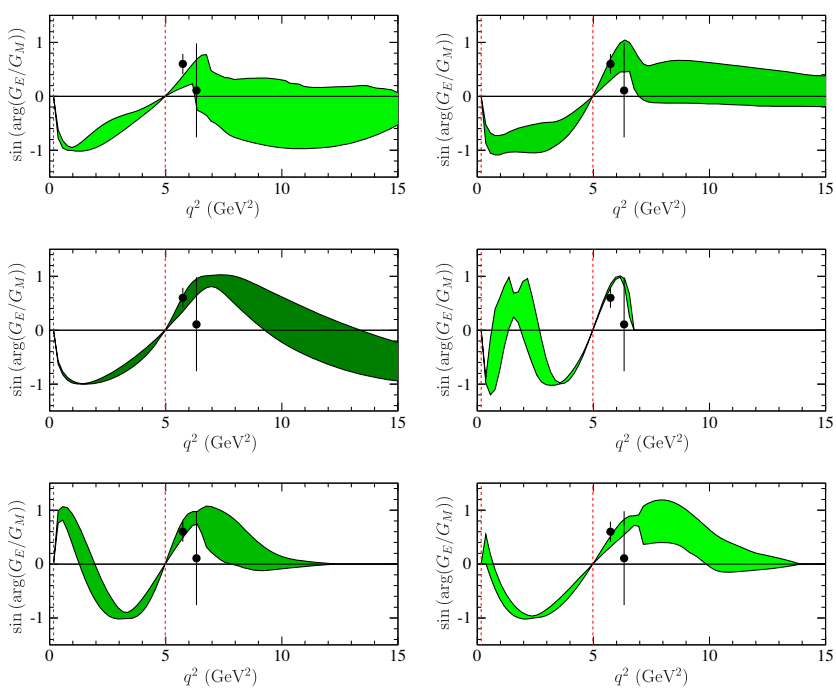

FIG. 10. Sinus of the phase of the FF ratio with: $\left(N_{\text {th }}, N_{\text {asy }}\right)=$ $(-1,0)$ in the upper-left position, $\left(N_{\text {th }}, N_{\text {asy }}\right)=(-1,1)$ in position right-up, $\left(N_{\text {th }}, N_{\text {asy }}\right)=(-1,2)$ in the middle-left position, $\left(N_{\text {th }}, N_{\text {asy }}\right)=(-1,3)$ in middle-right position, $\left(N_{\text {th }}, N_{\text {asy }}\right)=$ $(0,3)$ in the lower-left position, $\left(N_{\text {th }}, N_{\text {asy }}\right)=(1,3)$ in lower-right position. The color intensity of the error bands is proportional to the occurrence probability of the corresponding pair $\left(N_{\text {th }}, N_{\text {asy }}\right)-$ a darker color indicates an higher probability. The red dashed lines indicate the theoretical and the physical thresholds.

$$
\left\langle r_{E}\right\rangle^{2}=\left.6 \frac{d G_{E}\left(q^{2}\right)}{d q^{2}}\right|_{q^{2}=0},
$$

i.e., it is proportional to the first derivative of the electric FF with respect to the four-momentum squared at $q^{2}=0$. The definition follows from the fact that in the Breit frame, being no energy exchange between electron and baryon, the four-momentum $q$ is purely spacelike, i.e., $q=(0, \vec{q})$, then the electric FF represents the Fourier transform of the spacial charge distribution of the baryon.

It is interesting to notice that in the case of a neutral baryon with nonvanishing magnetic moment $\mu$, so that it has electric and magnetic FFs normalized as

$$
G_{E}(0)=0, \quad G_{M}(0)=\mu \neq 0,
$$

the charge radius can be defined in terms of the first derivative of the ratio $R\left(q^{2}\right)=G_{E}\left(q^{2}\right) / G_{M}\left(q^{2}\right)$ always at $q^{2}=0$. In fact

$$
\begin{aligned}
\left.\frac{d R\left(q^{2}\right)}{d q^{2}}\right|_{q^{2}=0}= & \frac{1}{G_{M}\left(q^{2}\right)}\left(\frac{d G_{E}\left(q^{2}\right)}{d q^{2}}\right. \\
& \left.-\frac{G_{E}\left(q^{2}\right)}{G_{M}\left(q^{2}\right)} \frac{d G_{M}\left(q^{2}\right)}{d q^{2}}\right)\left.\right|_{q^{2}=0}, \\
= & \left.\left(\frac{1}{G_{M}\left(q^{2}\right)} \frac{d G_{E}\left(q^{2}\right)}{d q^{2}}\right)\right|_{q^{2}=0}=\frac{1}{\mu} \frac{\left\langle r_{E}\right\rangle^{2}}{6},
\end{aligned}
$$


it follows that

$$
\left\langle r_{E}\right\rangle^{2}=\left.6 \mu \frac{d R\left(q^{2}\right)}{d q^{2}}\right|_{q^{2}=0} .
$$

On the other hand, a DR for the first derivative of $R\left(q^{2}\right)$ can be obtained from that of Eq. (A1), in particular with $q^{2}<q_{\mathrm{th}}^{2}$

$$
\frac{d R\left(q^{2}\right)}{d q^{2}}=\frac{1}{\pi} \int_{q_{\mathrm{th}}^{2}}^{\infty} \frac{\operatorname{Im}(R(s))}{\left(s-q^{2}\right)^{2}} d s,
$$

when evaluated at $q^{2}=0$ gives

$$
\left.\frac{d R\left(q^{2}\right)}{d q^{2}}\right|_{q^{2}=0}=\frac{1}{\pi} \int_{q_{\mathrm{th}}^{2}}^{\infty} \frac{\operatorname{Im}(R(s))}{s^{2}} d s
$$

By considering the parametrization of Eq. (12) this derivative can be expressed directly in terms of the free parameters of the set $\left\{\vec{C}=\left(C_{0}, C_{1}, \ldots, C_{N}\right), q_{\text {asy }}^{2}\right\}$ as follows:

$$
\left.\frac{d R\left(q^{2}\right)}{d q^{2}}\right|_{q^{2}=0}=\frac{1}{\pi \Delta q^{2}} \sum_{j=0}^{N} C_{j} \int_{-1}^{1} \frac{T_{j}(x)}{\left(x+1+q_{\mathrm{th}}^{2} / \Delta q^{2}\right)^{2}} d x,
$$

where $\Delta q^{2}=\left(q_{\text {asy }}^{2}-q_{\mathrm{th}}^{2}\right) / 2$ and the expression of the $x$ variable as a function of $q^{2}$ is given in Eq. (13).

The magnetic moment of the $\Lambda$ baryon is $\mu_{\Lambda}=$ $-0.613 \pm 0.004 \mu_{N}$ [12], and the squared charge radius can be obtained as

$$
\left\langle r_{E}^{\Lambda}\right\rangle^{2}=\frac{6 \mu_{\Lambda}}{\pi \Delta q^{2}} \sum_{j=0}^{N} C_{j} \int_{-1}^{1} \frac{T_{j}(x)}{\left(x+1+q_{\mathrm{th}}^{2} / \Delta q^{2}\right)^{2}} d x .
$$

Depending on the set of free parameters, i.e., from the analysis results this quantity can be both positive and negative. The best known particle with a negative squared charge radius is the neutron. Such a feature can be explained by considering the electric charge spatial distribution of the neutron due to the valence quarks dislocation, which, assuming the spherical symmetry, can be further simplified in terms of their mean distance from the center of the neutron. The squared charge radius will be negative when the two down valence quarks, bearing the negative charge, lie at a mean distance from the neutron center larger than the distance of the positively charged up quark.

This unbalanced distribution of charge within the volume of the neutron gives rise to the small negative squared charge radius $\left\langle r_{E}^{n}\right\rangle^{2}=-0.1161 \pm 0.0022 \mathrm{fm}^{2}$ [12]. In order to have a better understanding of the linear extension of the baryon we define a normalized charge radius by taking the square root of the modulus of the squared charge radius to which we assign the original sign, i.e.,

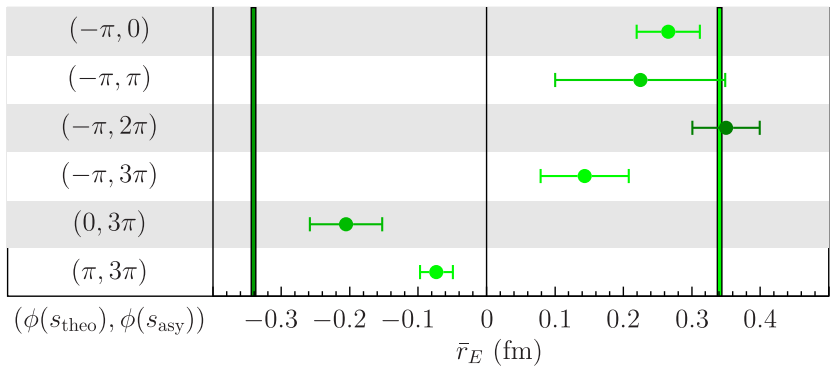

FIG. 11. The points represent the normalized charge radii obtained in the six cases under consideration, whose $\left(N_{\text {th }}, N_{\text {asy }}\right)$ pairs are reported on the left-hand side of the picture. Here, the intensity of the green color is proportional to the probability of the occurrence of each $\left(N_{\text {th }}, N_{\text {asy }}\right)$ pair. For comparison, the symmetric vertical green bars indicate the negative normalized neutron charge radius, on the left, and its reflection on the right.

$$
\bar{r}_{E}=\operatorname{Sign}\left[\left\langle r_{E}\right\rangle^{2}\right] \sqrt{\left|\left\langle r_{E}\right\rangle^{2}\right|} .
$$

Following this definition, the normalized charge radius of the neutron is $\bar{r}_{N}^{n}=-0.3407 \pm 0.0032 \mathrm{fm}$.

Figure 11 shows the quite different values of the normalized charge radius of the $\Lambda$ baryon obtained in this study, in comparison with that of the neutron, which is represented by the vertical dark green bar. For comparison, we also report its positive opposite, the light-green bar on the right hand side of Fig. 11.

The heuristic interpretation of this result is that, while in the case of neutron the two down quarks orbit at a distance that in average is larger than that of the up quark, giving an overall effect of a negative squared charge radius, for the $\Lambda$ baryon, in the most probable case with $\left(N_{\text {th }}, N_{\text {asy }}\right)=$ $(-1,2)$, see Table I, the configuration is the opposite. In fact, the negative charge, carried by the down and the heavy strange quarks, is mainly concentrated at small distances, resulting in a positive squared charge radius.

On the other hand, the second most probable result, corresponding to $\left(N_{\text {th }}, N_{\text {asy }}\right)=(0,3)$ and occurring with a probability of about half of that of the case with $\left(N_{\text {th }}, N_{\text {asy }}\right)=(-1,2)$, corresponds to a negative squared charge radius compatible with the that of the neutron.

Despite the powerful role played by the theoretical approach in establishing a set of reliable conditions which restrict the choice of possible configurations, the present discussion on the radius points to the need of further experimental investigation.

\section{CONCLUSION}

In order to study the $\Lambda$ baryon electromagnetic FFs, we have defined a phenomenological approach, which is based on first principles as analyticity and optical theorem, that 
have been implemented by means of the powerful mathematical tool of DRs.

The first and unique precise experimental data of the phase of the FF ratio $G_{E}^{\Lambda} / G_{M}^{\Lambda}$, obtained by the BESIII Collaboration [7], have been analyzed together with those on its moduli to gain a deeper knowledge of the dynamics which rule the electromagnetic coupling between the photon and the $\Lambda \bar{\Lambda}$ system in the timelike region, as well as the spacelike counterpart, i.e., the amplitude of the radiative virtual conversion $\Lambda(\bar{\Lambda}) \rightarrow \gamma \bar{\Lambda}(\Lambda)$.

This is, actually, the key point of our study; the connection between the timelike and the spacelike behaviors of the $\Lambda$ FFs. In particular, we take advantage of the fact, formalized by the Levinson's theorem [9], that the variation of the phase as a function of $q^{2}$ in the timelike interval, going from the theoretical threshold $q_{\mathrm{th}}^{2}$ up to infinity, is directly connected to the number of zeros of the electric FF $G_{E}^{\Lambda}$ in the spacelike region. This kinematic region, characterized by negative values of $q^{2}$, is not experimentally accessible for the $\Lambda$ baryon as well as for all other heavier baryons because their instability prevents scattering experiments with electron or muon beams.

The connection between timelike and spacelike behaviors provided by this dispersive approach represents a unique possibility to investigate the FFs at negative $q^{2}$ and then gain information on dynamical and static properties of the baryons. In more detail, concerning the phase, we find that in each of the six cases, reported in Table I, which, in the framework of our phenomenological description, are compatible with the exiguous set of available data, there is always a nonvanishing phase variation, i.e.,

$$
\Delta \phi=\phi(\infty)-\phi\left(q_{\mathrm{th}}^{2}\right)=\Delta N \pi=\left(N_{\mathrm{asy}}-N_{\mathrm{th}}\right) \pi \geq \pi .
$$

This means that the electric FF has at least one zero in the $q^{2}$ region below the theoretical threshold $q_{\mathrm{th}}^{2}$ and confirms the timelike $\leftrightarrow$ spacelike connection. In fact, the electric $\mathrm{FF}$, since the $\Lambda$ is a neutral baryon, must be normalized to zero at $q^{2}=0$.

The fact that the most probable solutions, see Table I, are those with $\Delta \phi=3 \pi$, with $\left(N_{\text {th }}, N_{\text {asy }}\right)=(-1,2)$ and $(0,3)$, represents a clear indication that $G_{E}^{\Lambda}$ has additional zeros. Such an eventuality, if confirmed by further studies and more exhaustive experimental investigations, would be a quite interesting feature that could be interpreted in terms of charge distribution and hence of the dynamical mechanism underlying the electromagnetic coupling of the $\Lambda$ baryons. The presence of unexpected spacelike zeros for the proton electric FF is object of a deep theoretical and experimental investigation since the years 2000 at MIT Bates and at Jefferson Lab (see Ref. [20] and references therein). Here the first measurements of the proton FF ratio $G_{E}^{p} / G_{M}^{p}$ have been performed by exploiting, for the first time, the polarization-transfer technique [21,22], obtaining results compatible with the presence of a zero for the ratio, and hence for $G_{E}^{p}$, lying at $q^{2} \sim-10 \mathrm{GeV}^{2}$. The results of the JLab-GEp Collaboration give only a hint for a spacelike zero, as they show a monotonic decrease of the proton FF ratio, which when extrapolated, would cross zero. The planned experiments at Jefferson Lab [23], by extending the kinematical region up to $q^{2}=-15 \mathrm{GeV}^{2}$, will answer this question. Once again, a spacelike zero for the proton electric FF determines the timelike behavior of the phase of the ratio $G_{E}^{p} / G_{M}^{p}$, which should have an increase of $\pi$ radians from the theoretical up to the asymptotic threshold. However, since the proton is a stable baryon, the only possibility of obtaining its polarization vector and hence to gain information on the $G_{E}^{p} / G_{M}^{p}$ phase, relies on a direct measurement of the polarization itself. Unfortunately, there are neither running nor planned future experiments able to perform this kind of measurements in the timelike region. Finally, we discussed the possibility of observing the determination of the phase, despite the fact that the measured observable, i.e., the sinus of the phase, does not contain this information. This severe limitation can be overcome by studying the $q^{2}$ evolution of the sinus of the phase in connection with the modulus of the FF ratio, in the framework of our dispersive procedure. In fact, as pointed out in Sec. VIII B, assuming that the ratio $R\left(q^{2}\right)=$ $G_{E}^{\Lambda}\left(q^{2}\right) / G_{M}^{\Lambda}\left(q^{2}\right)$ is an analytic function in the whole $q^{2}$ complex plane, with the branch cut $\left(q_{\mathrm{th}}^{2}, \infty\right)$, which means that the magnetic FF has no zeros in this domain, the phase can only increase as $q^{2}$ runs from the theoretical, $q_{\mathrm{th}}^{2}$, up to the asymptotic threshold $q_{\text {asy }}^{2}$.

As an example, we can consider a phase increasing from the multiple $N_{\text {th }}$ of $\pi$ radians, at $q_{\mathrm{th}}^{2}$, to the multiple $N_{\text {asy }}$, at $q_{\text {asy }}^{2}$, as shown in the upper panel of Fig. 9, so that $\left(N_{\text {th }}, N_{\text {asy }}\right)=(0,4)$. Such a phase corresponds to an oscillating behavior of the sinus, which starting and ending up at zero, undertakes a number of oscillations equal to $\Delta N=N_{\text {asy }}-N_{\text {th }}$. Here an oscillation is defined as the portion of the sinus function passing through either a maximum +1 or a minimum -1 , included between two consecutive nodes. The lower panel of Fig. 9 shows the four oscillations, passing through two maxima and two minima, undertaken by the sinus of the phase, shown in the upper panel, which increases from zero up to $4 \pi$ radians.

The actual experimental situation, even though is characterized by only one precise datum on the phase provided by the BESIII Collaboration, $\sin \left(\phi_{\text {BESIII }}\right)=0.60 \pm 0.19$ at $\sqrt{q^{2}}=2.396 \mathrm{GeV}$, appears quite promising. Indeed, this value of the sinus significantly different from zero is a clear indication that the phase at the corresponding $q^{2}$ is still increasing, and hence that the asymptotic threshold has been not yet reached.

In other words, the unique BESIII datum is sufficient to indicate that there is a non-negligible probability that, by exploring a wider $q^{2}$ region, more oscillations could be seen. The knowledge of the asymptotic value of the phase, 
i.e., the number of oscillations of its sinus would allow us to know how many zeros the electric FF has in the experimentally inaccessible spacelike region. This is a crucial information on the internal structure of the $\Lambda$ baryon.

Finally, a second precise determination of the sinus of the phase at a different $q^{2}$, not too far from that of the BESIII datum, would allow us to establish if the sinus at $\sqrt{q^{2}}=$ $2.396 \mathrm{GeV}$ is either increasing or decreasing. While in the latter case we could expect that the phase is approaching its asymptotic threshold and hence what we are measuring is just the last oscillation of its sinus. In the former case, i.e., if the sinus is increasing we have to expect a further complete oscillation.

\section{ACKNOWLEDGMENTS}

It is a privilege to acknowledge Rinaldo Baldini Ferroli for being an invaluable and boundless source of brilliant new ideas which pour out from any conversation with him. This work was supported in part by the STRONG-2020 project of the European Union's Horizon 2020 research and innovation program under Grant Agreement No. 824093.

\section{APPENDIX A: DISPERSION RELATIONS}

The most powerful analytic-continuation technique, especially suitable for FFs, is represented by the so-called DRs [24]. The feature of being analytically continued in an extended domain containing the original one represents a crucial property of analytic functions which is grounded on the principle of their uniqueness [8]. There are different forms of DRs. In particular, by considering an analytic and multivalued function $f(z)$, defined in the domain $C=\left\{z: z \notin\left(x_{0}, \infty\right)\right\}$, i.e., in the whole $z$-complex plane with the real branch cut $\left(x_{0}, \infty\right) \subset \mathbb{R}$ and the following three properties:

(i) $f(z) \in \mathbb{R}, \quad \forall z \in C \cap \mathbb{R}$, i.e., the function is real on the portion of real axis contained in its domain;

(ii) $f(z)=o(1 / \ln (|z|))$ as $z \rightarrow \infty$, i.e., the function is an infinitesimal of higher order than $1 / \ln (|z|)$ as $z$ diverges;

(iii) $f(z) \underset{z \rightarrow x_{0}}{\propto}\left(z-x_{0}\right)^{\sigma}$, with $\operatorname{Re}(\sigma)>-1$;

the DR for the imaginary part is

$$
f(z)=\frac{1}{\pi} \int_{x_{0}}^{\infty} \frac{\operatorname{Im}(f(x))}{x-z} d x, \quad \forall z \in C,
$$

where it is understood that the imaginary part is evaluated on the upper edge of the branch cut. If the asymptotic behavior is not the one required at the second item of the previous list, DRs with subtractions have to be used.

More precisely, the DR for the imaginary part subtracted $n$-times at the real point $x_{1} \in C$, i.e., $x_{1}<x_{0}$, is

$$
\begin{aligned}
f(z)= & \sum_{k=0}^{n-1} \frac{f^{(k)}\left(x_{1}\right)}{k !}\left(z-x_{1}\right)^{k} \\
& +\frac{\left(z-x_{1}\right)^{n}}{\pi} \int_{x_{0}}^{\infty} \frac{\operatorname{Im}(f(x))}{\left(x-x_{1}\right)^{n}(x-z)} d x, \quad \forall z \in C,
\end{aligned}
$$

where $f^{(k)}\left(z^{\prime}\right)$ indicates the $k$ th derivative of the function $f(z)$ evaluated at $z=z^{\prime}$. Such a form of DR is obtained by writing the DR for the imaginary part of Eq. (A1) for the function

$$
g(z)=\frac{f(z)-\sum_{k=0}^{n-1} \frac{f^{(k)}\left(x_{1}\right)}{k !}\left(z-x_{1}\right)^{k}}{\left(z-x_{1}\right)^{n}},
$$

which has the same analytic properties of the function $f(z)$, with the exception of the asymptotic behavior. Indeed, the limits of the two functions $f(z)$ and $g(z)$, as $z$ diverges, differ by the power $z^{n}$, i.e., $g(z)=\mathcal{O}\left(f(z) / z^{n}\right)$, as $z \rightarrow \infty$. It follows that if the function $f(z)$ had a pole of order $m$ at infinity, so that $f(z)=\mathcal{O}\left(z^{m}\right)$ as $z \rightarrow \infty$, then it would be sufficient to make $n=m+1$ subtractions in order to have $g(z)=\mathcal{O}\left(z^{-1}\right)=o(1 / \ln (|z|))$. The cost of using the $n$ subtracted DR of Eq. (A2) is the knowledge of the values of the first $n$ derivatives (from the zero derivative, the function itself, up to the $(n-1)^{\text {th }}$ of the function in the point of subtraction, $z=x_{1}$.

The simplest form of $n$-subtracted DR is the one with only one subtraction. In this case $n=1$ and the expression of Eq. (A2) becomes

$$
f(z)=f\left(x_{1}\right)+\frac{z-x_{1}}{\pi} \int_{x_{0}}^{\infty} \frac{\operatorname{Im}(f(x))}{\left(x-x_{1}\right)(x-z)} d x,
$$

$\forall z \in C$. The one subtracted DR has to be used for those functions having a finite, non-null asymptotic value so that $f(z)=\mathcal{O}(1)$ as $z \rightarrow \infty$. In this case, only the value of the function at the subtraction point $z=x_{1}$ has to be known. By means of the well-known Sokhotski-Plemelj formula [25]

$\lim _{\epsilon \rightarrow 0^{+}} \int_{-\infty}^{\infty} \frac{f\left(x^{\prime}\right)}{x^{\prime}-x-i \epsilon} d x^{\prime}=\operatorname{Pr} \int_{-\infty}^{\infty} \frac{f\left(x^{\prime}\right)}{x^{\prime}-x} d x^{\prime}+i \pi f(x)$,

where the symbol $\operatorname{Pr} \int$ stands for the Cauchy principal value integral [8] and the function $f(z)$ is analytic in the infinite rectangle $R=\{z: \operatorname{Im}(z)=y \in(\epsilon / 2,3 \epsilon / 2)\} \subset\{z: \operatorname{Im}(z)=$ $y>0\}$, belonging to the upper-half $z$-complex plane, and such that 


$$
\lim _{x \rightarrow \pm \infty} f(x+i y)=0, \quad \forall y \in(\epsilon / 2,3 \epsilon / 2)
$$

the DR for the imaginary part of Eq. (A1) can be evaluated also on the upper edge of the real branch cut $\left(x_{0}, \infty\right)$. In particular, by taking $z=x+i \epsilon$, with $x>x_{0}$ and $\epsilon \rightarrow 0^{+}$, using the Sokhotski-Plemelj formula of Eq. (A4) and showing explicitly the infinitesimal and positive imaginary part $\epsilon$ the DR can be written as

$$
\begin{aligned}
f(x+i \epsilon) & =\frac{1}{\pi} \int_{x_{0}}^{\infty} \frac{\operatorname{Im}\left(f\left(x^{\prime}+i \epsilon\right)\right)}{x^{\prime}-x-i \epsilon} d x^{\prime} \\
& =\frac{1}{\pi} \int_{-\infty}^{\infty} \frac{\operatorname{Im}\left(f\left(x^{\prime}+i \epsilon\right)\right)}{x^{\prime}-x-i \epsilon} d x^{\prime} \\
& =\frac{\operatorname{Pr}}{\pi} \int_{-\infty}^{\infty} \frac{\operatorname{Im}\left(f\left(x^{\prime}+i \epsilon\right)\right)}{x^{\prime}-x} d x^{\prime}+i \operatorname{Im}(f(x+i \epsilon)) .
\end{aligned}
$$

The extension of the integration interval up to the whole real axis does not imply any additional contribution because the imaginary part of the function $f(z)$ is null for all $z \in\left(-\infty, x_{0}\right]$. By adding $-i \operatorname{Im}(f(x+i \epsilon))$ to both sides of the previous equation one obtains the so-called DR for the real part, that gives the real part of the function $f(z)$ on the upper edge of the branch cut, i.e.,

$$
\operatorname{Re}(f(x))=\frac{\operatorname{Pr}}{\pi} \int_{-\infty}^{\infty} \frac{\operatorname{Im}\left(f\left(x^{\prime}\right)\right)}{x^{\prime}-x} d x^{\prime}
$$

where, as in Eq. (A1), the infinitesimal imaginary part has to be understood assuming that both imaginary and real part are always evaluated on the upper edge of the branch cut. ${ }^{3}$

Finally, the DR for the real part can be also subtracted; the expression with $n$ subtractions at $z=x_{1}<x_{0}$ follows similarly to the derivation of Eq. (A2). In particular, $\forall x>x_{0}$, we have

$$
\begin{aligned}
\operatorname{Re}(f(x))= & \sum_{k=0}^{n-1} \frac{f^{(k)}\left(x_{1}\right)}{k !}\left(x-x_{1}\right)^{k} \\
& +\frac{\left(x-x_{1}\right)^{n}}{\pi} \operatorname{Pr} \int_{x_{0}}^{\infty} \frac{\operatorname{Im}\left(f\left(x^{\prime}\right)\right)}{\left(x^{\prime}-x_{1}\right)^{n}\left(x^{\prime}-x\right)} d x^{\prime}
\end{aligned}
$$

\footnotetext{
${ }^{3}$ Notice that, as a consequence of the Schwarz reflection principle, while the real part of the function assumes the same value on the upper and lower edge of the branch cut, the imaginary part has a discontinuity, by changing the sign, i.e., $\forall x>x_{0}$,
}

$$
\begin{aligned}
& \lim _{\epsilon \rightarrow 0^{+}} \operatorname{Re}(f(x+i \epsilon))=\lim _{\epsilon \rightarrow 0^{+}} \operatorname{Re}(f(x-i \epsilon)), \\
& \lim _{\epsilon \rightarrow 0^{+}} \operatorname{Im}(f(x+i \epsilon))=-\lim _{\epsilon \rightarrow 0^{+}} \operatorname{Im}(f(x-i \epsilon)) .
\end{aligned}
$$

which in the simplest case of only one subtraction becomes

$$
\operatorname{Re}(f(x))=f\left(x_{1}\right)+\frac{x-x_{1}}{\pi} \operatorname{Pr} \int_{x_{0}}^{\infty} \frac{\operatorname{Im}\left(f\left(x^{\prime}\right)\right)}{\left(x^{\prime}-x_{1}\right)\left(x^{\prime}-x\right)} d x^{\prime} .
$$

\section{APPENDIX B: THE MINIMIZATION PROCEDURE}

The error bands have been obtained by means of a Monte Carlo technique consisting of the following steps.

(a) By Gaussian oscillations of the original one, $S$ sets of data have been randomly generated.

(b) The minimization procedure has been performed on each of the $S$ sets of data by obtaining $S$ sets of parameters $\left\{\left(C_{0, j}, C_{1, j}, \ldots, C_{N, j}\right), q_{\text {asy }, j}^{2}\right\}_{j=1}^{S}$ and corresponding curves, e.g., the modulus and phase of the ratio $\left\{\left|G_{E}^{\Lambda} / G_{M}^{\Lambda}\right|_{j}, \arg \left(G_{E}^{\Lambda} / G_{M}^{\Lambda}\right)_{j}\right\}_{j=1}^{S}$.

(c) The best values of the parameters, their standard deviations, as well as the error bands have been obtained by means of the standard statistical techniques.

For instance, the lower and upper limits of the error band for the modulus $R \equiv\left|G_{E}^{\Lambda} / G_{M}^{\Lambda}\right|$, at a given $q^{2}$ are

$$
R\left(q^{2}\right)^{ \pm}=\overline{R\left(q^{2}\right)} \pm \delta\left[R\left(q^{2}\right)\right],
$$

with the mean value and standard deviation

$$
\begin{aligned}
\overline{R\left(q^{2}\right)} & =\frac{\sum_{j=1}^{S} R_{j}\left(q^{2}\right)}{S}, \\
\delta\left[R\left(q^{2}\right)\right] & =\sqrt{\frac{\sum_{j=1}^{S}\left(R_{j}\left(q^{2}\right)-\overline{R\left(q^{2}\right)}\right)^{2}}{S-1}},
\end{aligned}
$$

where $R_{j}\left(q^{2}\right)=\left|G_{E}^{\Lambda}\left(q^{2}\right) / G_{M}^{\Lambda}\left(q^{2}\right)\right|_{j}$ represents the modulus of the ratio at $q^{2}$ which has been obtained by using the $j$ th set of parameters $\left\{\left(C_{0, j}, C_{1, j}, \ldots, C_{N, j}\right), q_{\text {asy }, j}^{2}\right\}$, with $j=1,2, \ldots, S$.

The same procedure has been used to obtain the values of the parameters reported in Table II, i.e.,

$$
C_{k}=\bar{C}_{k} \pm \delta\left[C_{k}\right], \quad q_{\text {asy }}^{2}=\overline{q_{\text {asy }}^{2}} \pm \delta\left[q_{\text {asy }}^{2}\right]
$$

where the mean values and standard deviations are

$$
\begin{array}{cc}
\bar{C}_{k}=\frac{\sum_{j=1}^{S} C_{k, j}}{S}, \quad \delta\left[C_{k}\right]=\sqrt{\frac{\sum_{k=1}^{S}\left(C_{k, j}-\bar{C}_{k}\right)^{2}}{S-1},} \\
\overline{q_{\text {asy }}^{2}}=\frac{\sum_{j=1}^{S} q_{\text {asy }, j}^{2}}{S}, & \delta\left[q_{\text {asy }}^{2}\right]=\sqrt{\frac{\sum_{k=1}^{S}\left(q_{\text {asy }, j}^{2}-\overline{q_{\text {asy }}^{2}}\right)^{2}}{S-1}},
\end{array}
$$

with $k=0,1, \ldots, 5$. In fact, as discussed in Sec. VII, we have chosen $N=5$ as the maximum degree of Chebyshev's 
polynomials used to parametrize the imaginary part of Eq. (12). It follows that by considering the first principles constraints represented by the three zeros that this imaginary part has to have at the so-called theoretical, physical and asymptotic thresholds, $q_{\mathrm{th}}^{2}, q_{\mathrm{phy}}^{2}$, and $q_{\text {asy }}^{2}$, respectively, there are $N-1=4$ free parameters. In more detail, three out of the six Chebyshev's coefficients can be obtained as combinations of the others, as specified in Table II. In the case under consideration there is only one degree of freedom. In general, the number of degrees of freedom, $N_{\text {DoF }}$, is given by

$$
N_{\text {DoF }}=\underbrace{N_{\text {Data }}}_{M+P=5}-\underbrace{N_{\text {Parameters }}}_{N-1=4}=1,
$$

where $M=3$ and $P=2$, see Secs. IV and VII, are the numbers of data points provided by BABAR and BESIII experiments, respectively. So the definition in Eq. (15) and shown in the lower panel of Fig. 5 is also the normalized $\chi^{2}$, which coincides with the non-normalized one.

\section{APPENDIX C: SCHEMES OF QCD CORRECTIONS}

For illustration, we consider the following two examples where the implementation of QCD corrections leads to different asymptotic behaviors for the FF ratio $R\left(q^{2}\right)$.

(1) From their analysis, performed in the framework of perturbative QCD, A. V. Belitsky, X. Ji and F. Yuan [26] obtained for the ratio of the Dirac, $F_{1}$, and Pauli $\mathrm{FF}, F_{2}$, at the logarithmic accuracy, the asymptotic behavior

$$
\frac{F_{2}\left(q^{2}\right)}{F_{1}\left(q^{2}\right)} \underset{\left|q^{2}\right| \rightarrow \infty}{\propto}-\frac{\ln ^{2}\left(q^{2} / \Lambda_{\text {soft }}^{2}\right)}{q^{2}},
$$

where $\Lambda_{\text {soft }}$ is what they called a soft scale related to the size of the baryon, ${ }^{4}$ that is of the order of a few hundreds of $\mathrm{MeV}$. In this case the ratio diverges like $\ln ^{2}\left(\left|q^{2}\right|\right)$ as $\left|q^{2}\right| \rightarrow \infty$. By exploiting the well-known expressions of the electric and magnetic FFs in terms of the Dirac and Pauli ones, i.e.,

\footnotetext{
${ }^{4}$ In natural units $1 \mathrm{fm}^{-1} \simeq 197 \mathrm{MeV}$.
}

$$
\begin{aligned}
R\left(q^{2}\right) & =\frac{G_{E}^{\Lambda}\left(q^{2}\right)}{G_{M}^{\Lambda}\left(q^{2}\right)} \\
& =\frac{F_{1}^{\Lambda}\left(q^{2}\right)+\frac{q^{2}}{4 M_{\Lambda}^{2}} F_{2}^{\Lambda}\left(q^{2}\right)}{F_{1}^{\Lambda}\left(q^{2}\right)+F_{2}^{\Lambda}\left(q^{2}\right)} \\
& =\frac{1+\frac{q^{2}}{4 M_{\Lambda}^{2}} \frac{F_{2}^{\Lambda}\left(q^{2}\right)}{F_{1}^{\Lambda}\left(q^{2}\right)}}{1+\frac{F_{2}^{\Lambda}\left(q^{2}\right)}{F_{1}^{\Lambda}\left(q^{2}\right)}}
\end{aligned}
$$

and using the result of Eq. (C1) for the ratio $F_{2}^{\Lambda} / F_{1}^{\Lambda}$, we have

$$
R\left(q^{2}\right) \underset{\left|q^{2}\right| \rightarrow \infty}{\propto}-\frac{\ln ^{2}\left(q^{2} / \Lambda_{\text {soft }}^{2}\right)}{4 M_{\Lambda}^{2}}
$$

(2) The second example is due to M. Gari and W. Krumpelmann [27-29] who proposed to consider QCD correction by substituting $q^{2}$ with

$$
q^{2} \rightarrow \tilde{q}^{2}=q^{2} \frac{\ln \left(\Lambda_{2}^{2}-q^{2}\right)-\ln \left(\Lambda_{\mathrm{QCD}}^{2}\right)}{\ln \left(\Lambda_{2}^{2}\right)-\ln \left(\Lambda_{\mathrm{QCD}}^{2}\right)},
$$

where $\Lambda_{\mathrm{QCD}}=0.3 \mathrm{GeV}$, while $\Lambda_{2}$, being of the order of a few $\mathrm{GeV}$, represents the threshold from which FFs start to follow the perturbative QCD power-law. In this case the ratio of the Dirac and Pauli FFs scales as

$$
\frac{F_{2}\left(q^{2}\right)}{F_{1}\left(q^{2}\right)} \underset{\left|q^{2}\right| \rightarrow \infty}{\sim}-\frac{[\text { constant }]}{q^{2} \ln \left(q^{2} / \Lambda_{\mathrm{QCD}}^{2}\right)} .
$$

It follows that the ratio of FFs is asymptotically constant and equal to one, i.e.,

$$
R\left(q^{2}\right) \underset{\left|q^{2}\right| \rightarrow \infty}{\propto} \frac{1-\frac{[\text { constant }]}{4 M_{\Lambda}^{2} \ln \left(q^{2} / \Lambda_{\mathrm{QCD}}^{2}\right)}}{1-\frac{[\text { constant }]}{q^{2} \ln \left(q^{2} / \Lambda_{\mathrm{QCD}}^{2}\right)}} \underset{\left|q^{2}\right| \rightarrow \infty}{\rightarrow} 1,
$$

where we have used the expression of the ratio given in Eq. (C2).
[1] S. Pacetti, R. Baldini Ferroli, and E. Tomasi-Gustafsson, Phys. Rep. 550-551, 1 (2015).
[2] F. J. Ernst, R. G. Sachs, and K. C. Wali, Phys. Rev. 119, 1105 (1960). 
[3] M. P. Rekalo, Sov. J. Nucl. Phys. 1, 760 (1965).

[4] C. Adamuscin, E. A. Kuraev, E. Tomasi-Gustafsson, and F. E. Maas, Phys. Rev. C 75, 045205 (2007).

[5] A. Z. Dubnickova, S. Dubnička, and M. P. Rekalo, Nuovo Cimento A 109, 241 (1996).

[6] B. Aubert et al. (BABAR Collaboration), Phys. Rev. D 76, 092006 (2007).

[7] M. Ablikim et al. (BESIII Collaboration), Phys. Rev. Lett. 123, 122003 (2019).

[8] Lars V. Ahlfors, Complex Analysis: An Introduction to the Theory of Analytic Functions of One Complex Variable (McGraw-Hill Education, New York, 1979).

[9] N. Levinson, Kgl. Danske Videnskab. Selskab, Mat.-Fys. Medd. 25, 1 (1949).

[10] G. Hohler, E. Pietarinen, I. Sabba Stefanescu, F. Borkowski, G. G. Simon, V. H. Walther, and R. D. Wendling, Nucl. Phys. B114, 505 (1976).

[11] G. Hohler and E. Pietarinen, Nucl. Phys. B95, 210 (1975).

[12] P. A. Zyla et al. (Particle Data Group), Prog. Theor. Exp. Phys. (2020), 083C01.

[13] See for instance R. Karplus, C. M. Sommerfield, and E. H. Wichmann, Phys. Rev. 111, 1187 (1958).

[14] See for instance V. Bernard, N. Kaiser, and U. G. Meissner, Nucl. Phys. A611, 429 (1996).

[15] J. M. Alarcón, A. N. Hiller Blin, M. J. Vicente Vacas, and C. Weiss, Nucl. Phys. A964, 18 (2017).

[16] V. A. Matveev, R. M. Muradyan, and A. N. Tavkhelidze, Teor. Mat. Fiz. 15, 332 (1973).

[17] S. J. Brodsky and G. R. Farrar, Phys. Rev. Lett. 31, 1153(1973).

[18] M. Abramowitz and I. A. Stegun, Handbook of Mathematical Functions with Formulas, Graphs, and Mathematical
Tables, Applied Mathematics Series Vol. 55 (Ninth reprint with additional corrections of tenth original printing with corrections; first ed.), Washington D. C., 1972.

[19] C. W. Groetsch, Inverse Problems in Mathematical Sciences (Vieweg, Braunschweig, 1993).

[20] A. J. R. Puckett, E. J. Brash, M. K. Jones, W. Luo, M. Meziane, L. Pentchev, C. F. Perdrisat, V. Punjabi, F. R. Wesselmann, A. Afanasev et al., Phys. Rev. C 96, 055203 (2017); 98, 019907(E) (2018).

[21] A. I. Akhiezer and M. P. Rekalo, Sov. Phys. Dokl. 13, 572 (1968).

[22] A. I. Akhiezer and M. P. Rekalo, Fiz. Elem. Chastits At. Yadra 4, 662 (1973).

[23] E. Brash, E. Cisbani, M. Jones, M. Khandaker, N. Liyanage, L. Pentchev, C. F. Perdrisat, V. Punjabi, and B. Wojtsekhowski, Jefferson Lab proposal 12-07-109, Large Acceptance Proton Form Factor Ratio Measurements up to 14.5 GeV $\mathrm{GeV}^{2}$ Using Recoil-Polarization Method, https:// userweb.jlab.org/bogdanw/gep_u.pdf.

[24] See for instance Jon Mathews and Robert Lee Walker, Mathematical Methods of Physics (W. A. Benjamin, 1970).

[25] See for instance V. S. Vladimirov, Equations of Mathematical Physics (M. Dekker, 1971).

[26] A. V. Belitsky, X. d. Ji, and F. Yuan, Phys. Rev. Lett. 91, 092003 (2003).

[27] M. Gari and W. Krumpelmann, Phys. Lett. 141B, 295 (1984).

[28] M. Gari and W. Krumpelmann, Z. Phys. A 322, 689 (1985).

[29] M. Gari and W. Krumpelmann, Phys. Lett. 173B, 10 (1986). 